\title{
THE
}

1981

\section{Quantum spin dynamics of the one-dimensional planar antiferromagnet}

Gerhard Müller

University of Rhode Island, gmuller@uri.edu

Harry Thomas

Marcos W. Puga

Hans Beck

Follow this and additional works at: https://digitalcommons.uri.edu/phys_facpubs

Terms of Use

All rights reserved under copyright.

\section{Citation/Publisher Attribution}

Müller, G., Thomas, H., Puga, M. W., \& Beck, H. (1981). Quantum spin dynamics of the one-dimensional planar antiferromagnet. J. Phys. C, 14, 3399-3422. doi: 10.1088/0022-3719/14/23/017

Available at: http://dx.doi.org/10.1088/0022-3719/14/23/017

This Article is brought to you for free and open access by the Physics at DigitalCommons@URI. It has been accepted for inclusion in Physics Faculty Publications by an authorized administrator of DigitalCommons@URI. For more information, please contact digitalcommons-group@uri.edu. 


\title{
Quantum spin dynamics of the one-dimensional planar antiferromagnet
}

\author{
Gerhard Müller, 1 Harry Thomas 1 Marcos W. Puga ${ }^{2}$ and Hans Beck ${ }^{2}$ \\ 1 Institut für Physik, Universität Basel, $\mathrm{CH}-4056$ Basel, Switzerland \\ 2 Institut de Physique de l'Université, CH-2000 Neuchâtel, Switzerland
}

\begin{abstract}
The $T=0$ dynamics of the one-dimensional $s=\frac{1}{2}$ planar anti ferromagnet is studied by an approach which consists of exact analytic calculations in the Bethe formalism and numerical finite-chain calculations on rings up to 10 spins. Our method makes use of well known critical exponents for the correlation functions and of exact sum rules. We obtain approximate analytic expressions for both the out-of-plane and the inplane dynamic structure factors, and for related quantities such as integrated intensities, susceptibilities and autocorrelation functions. The results are discussed in relation to possible experiments on quasi-1D magnetic compounds at low $T$. Our calculations make clear that the $T=0$ dynamic structure factors are dominated by two-parameter continua of excitations rather than by single branches of spin-waves as predicted by classical spin-wave theory. By varying the planar anisotropy the autocorrelation functions display interesting features in their long-time asymptotic behaviour, such as a crossover from a uniform power-law decay to an oscillatory decay and a crossover between oscillatory decays with different frequencies. We conjecture a possibility of approaching the classical limit $s=\infty$ starting from the quantum limit $s=\frac{1}{2}$. This provides a qualitative, and in some aspects even quantitative, understanding of the dynamical behaviour of $s>\frac{1}{2}$ systems in terms of a quantum approach.
\end{abstract}

\section{Introduction}

The number of available quasi-one-dimensional (ID) magnetic compounds is steadily increasing, conferring experimental relevance to more and more types of $1 \mathrm{D}$ spin model systems. This is particularly true for 1D antiferromagnets (AF) with nearest-neighbour (NN) exchange interactions including anisotropies. Experimental investigations of their static and dynamic properties are being performed with increasing accuracy, allowing the detection of more and more subtle effects. So far the interpretation of dynamical experimental data on quasi-1D AF systems has relied heavily on classical $(s \rightarrow \infty)$ or semiclassical $(s \gg 1)$ theories, although the actual spin quantum numbers involved are usually fairly small: $s=\frac{1}{2}, 1, \ldots, \frac{5}{2}$. Recent experiments at low temperatures indeed show substantial discrepancies from the predictions of linear spin-wave theory. These deviations are, of course, most pronounced for $s=\frac{1}{2}$ systems (Heilmann et al 1978, Groen et al 1980), but they are also observed in a system with $s=\frac{5}{2}$ (Heilmann et al 1979). Not surprisingly, at low $\mathrm{T}$ quantum effects are expected to be important. This is confirmed by a number of recent quantum approaches to the 1D spin-dynamics at $T=0$ (Luther and Peschel 1975, Mikeska 1975, Fogedby 1978, Vaidya and Tracy 1978, Fowler and Puga 1979, Müller et al 1979, Ishimura and Shiba 1980). Quantum effects are even more dramatic when a magnetic field is applied (Ishimura and Shiba 1977, Groen et al 1979, 1980, Müller et al 1981a, b).

In a recent study (Müller et al 1979, $1981 \mathrm{~b}$ ) we have investigated the $T=0$ dynamics of the isotropic $s=\frac{1}{2}$ Heisenberg AF. We have developed a new approach which does not involve the many-body techniques usually employed, but is based on exact analytic calculations in Bethe's formalism (Bethe 1931) combined with numerical finite-chain calculations (e.g. Müller and Beck 1978). We found that the $T=0$ dynamic structure factor $S_{\mu \mu}(q, \omega)$ is almost completely dominated by a two-parameter continuum of excitations rather than by a single branch as predicted by linear spin-wave theory (Anderson 1952). The result is in good agreement with low- $T$ neutron scattering data on CPC (Heilmann et al 1978) concerning excitation energies, lineshapes and integrated intensity. 
In this work we present an extension of the approach to the planar $s=\frac{1}{2} \mathrm{AF}$ (as defined below) along the following lines: (i) We use numerical finite-chain calculations and exact selection rules in order to select those excitations which contribute predominantly to the $T=0$ dynamics. (ii) Exact calculations using the Bethe ansatz allow us to identify these excitations as belonging to a special class of unbound states. We calculate their energies and density of states in the thermodynamic limit. (iii) We propose a simple method, based on a plausible assumption, by which the well known relation between the critical exponents of the Baxter model and exponents of the $s=\frac{1}{2} 1 \mathrm{D}$ spin system can be exploited in order to obtain matrix elements between the ground state and this special class of relevant excitations. This information is synthesised into an (approximate) analytic expression for the dynamic structure factor. In the limit of the $X Y$ AF this approach becomes exact for the out-of-plane fluctuations. For the other cases the accuracy of our approximation has been estimated to be high.

In Sec. 2 we introduce the model Hamiltonian, various observable quantities useful in connection with neutron scattering and NMR experiments, and an important sum rule for dynamic quantities. Section 3 gives a detailed account of our approach, resulting in analytic expressions for the out-of-plane (OP) and the in-plane (IP) dynamic structure factors. We discuss their implications concerning lineshapes and integrated intensities, and evaluate related quantities such as susceptibilities, static correlation functions, and autocorrelation functions. Section 4 deals with $s>\frac{1}{2}$ effects. We compare our $s=\frac{1}{2}$ results with the predictions of classical spin-wave theory, and we show a plausible method of approaching the classical limit starting from the complex behaviour for $s=\frac{1}{2}$.

\section{Model system and characteristic quantities}

The Hamiltonian of the 1D planar AF is given by

$$
\mathcal{H}=\sum_{l=1}^{N}\left[J_{\perp}\left(S_{l}^{x} S_{l+1}^{x}+S_{l}^{y} S_{l+1}^{y}\right)+J_{z} S_{l}^{z} S_{l+1}^{z}\right]
$$

with $0 \leq J_{z} \leq J_{\perp}$ and $s=\frac{1}{2}, 1, \frac{3}{2}, \ldots$ In the following we characterise the planar anisotropy by the dimensionless parameter

$$
\Delta=J_{z} / J_{\perp}, \quad 0 \leq \Delta \leq 1 .
$$

Throughout this paper energies and frequencies will be given in units of $J \equiv J_{\perp}$.

The symmetries of the Hamiltonian (2.1) include (i) invariance with respect to lattice translations (due to periodic boundary conditions): (ii) invariance with respect to rotations around the $z$ axis in spin space; (iii) for $\Delta=1$ only: complete rotational invariance in spin space. This allows for a characterisation of the eigenstates of (2.1) by the following quantum numbers:

(i) the wavenumber $q=(2 \pi / N) n, n=0, \pm 1, \pm 2, \ldots,+N / 2$;

(ii) the $z$-component of the total spin $S_{z}^{\mathrm{T}}=\sum_{l} S_{l}^{z}$,

(iii) for $\Delta=1$ : the magnitude of the total spin $S^{\mathrm{T}}\left(S^{\mathrm{T}}+1\right)=\left(\sum_{l} \mathbf{S}_{l}\right)^{2}$.

We are mainly interested in the following observable quantities characterising the zero-temperature properties of the spin chain:

(i) The dynamic structure factor

$$
S_{\mu \mu}(q, \omega) \equiv \sum_{R} \exp (-\mathrm{i} q R) \int_{-\infty}^{+\infty} \mathrm{d} t \mathrm{e}^{\mathrm{i} \omega t}\left\langle S_{l}^{\mu}(t) S_{l+R}^{\mu}\right\rangle, \quad \mu=x, y, z .
$$

This is directly related to the inelastic neutron scattering cross section (Marshall and Lovesey 1971). At $T=0$ it vanishes for $\omega<0$. The symmetry of (2.1) requires that the two IP components are identical: $S_{x x}(q, \omega)=S_{y y}(q, \omega)$. For $\Delta=1$ both are identical to the OP component $S_{z z}(q, \omega)$. Due to the reflection symmetry in real space all wavenumber-dependent properties are necessarily even functions of q. Hence, for simplicity we present in the following results only for $q \geq 01$

\footnotetext{
${ }^{1}$ It is convenient to introduce the wavenumber $\bar{q}=\pi-q$ which measures the deviation of $q$ from the Brillouin zone boundary of the quantum ground state, i,e. from the Bragg point of the classical Néel ground state of $(2,1)$.
} 
(ii) The static structure factor (integrated intensity)

$$
I_{\mu \mu}(q) \equiv \int_{0}^{\infty} \frac{\mathrm{d} \omega}{2 \pi} S_{\mu \mu}(q, \omega) .
$$

This is related to the quasielastic neutron scattering cross section.

(iii) The static correlation function $\left\langle S_{l}^{\mu} S_{l+R}^{\mu}\right\rangle$, which is the Fourier transform of $I_{\mu \mu}(q)$. Its asymptotic behaviour for large $R$ characterises the degree of ordering in the ground state.

(iv) The static $q$-dependent susceptibility $\chi_{\mu \mu}(q)$. At $T=0$ it is related to $S_{\mu \mu}(q, \omega)$ by the sum rule (Hohenberg and Brinkman 1974):

$$
\chi_{\mu \mu}(q)=2 \int_{0}^{\infty} \frac{\mathrm{d} \omega}{2 \pi} \omega^{-1} S_{\mu \mu}(q, \omega) .
$$

For the uniform susceptibility

$$
\chi_{\mu \mu}(q=0)=\left.\left(\mathrm{d} \sigma_{\mu} / \mathrm{d} h_{\mu}\right)\right|_{h_{\mu}=0}
$$

there are already some exact results available $\left(\sigma\right.$ is the magnetisation and $\mathbf{h}=g \mu_{\mathrm{B}} \mathbf{B} / J$ a reduced magnetic field).

(v) The autocorrelation function $\left\langle S_{l}^{\mu}(t) S_{l}^{\mu}\right\rangle$ and its Fourier transform

$$
F_{\mu \mu}(\omega) \equiv \int_{-\infty}^{+\infty} \mathrm{d} t \mathrm{e}^{\mathrm{i} \omega t}\left\langle S_{l}^{\mu}(t) S_{l}^{\mu}\right\rangle=N^{-1} \sum_{q} S_{\mu \mu}(q, \omega) .
$$

The low-frequency behaviour of $F_{\mu \mu}(\omega)$ is of particular experimental interest because it is directly related to proton spin-lattice relaxation rates measured on appropriate quasi-1D magnetic compounds (see e.g. Groen et al 1979, 1980).

There is an important sum rule relating the first frequency moment of $S_{\mu \mu}(q, \omega)$ to a static quantity which for the model system (2.1) can be expressed in terms of NN correlation functions (Hohenberg and Brinkman 1974)

$$
K_{\mu \mu}(q) \equiv \int_{0}^{\infty} \frac{\mathrm{d} \omega}{2 \pi} \omega S_{\mu \mu}(q, \omega)=-\frac{1}{2}\left\langle\left[\left[\mathcal{H}, S^{\mu}(q)\right], S^{\mu}(-q)\right]\right\rangle
$$

with

$$
S^{\mu}(q)=N^{-1 / 2} \sum_{l=1}^{N} \mathrm{e}^{\mathrm{i} q l} S_{l}^{\mu} .
$$

Irrespective of the value of the spin quantum number in $\mathcal{H}$, this quantity yields for $\mu=x, z$, respectively:

$$
\begin{aligned}
& K_{x x}(q)=-\left(J_{\perp} F_{x}+J_{z} F_{z}\right)+\left(J_{\perp} F_{z}+J_{z} F_{x}\right) \cos q, \\
& K_{z z}(q)=2 J_{\perp} F_{x}(1-\cos q),
\end{aligned}
$$

with $F_{\mu}=\left\langle S_{l}^{\mu} S_{l+1}^{\mu}\right\rangle$. At $T=0$ the $F_{\mu}$ in turn are related to the ground-state energy of $(2.1)$

$$
\epsilon_{\mathrm{G}}(\Delta) \equiv E_{\mathrm{G}} / N=J\left[2 F_{x}(\Delta)+\Delta F_{z}(\Delta)\right]
$$

The Feynman theorem (Feynman 1939) provides another relation between $\epsilon_{\mathrm{G}}$ and the $F_{\mu}$

$$
\partial \epsilon_{\mathrm{G}}(\Delta) / \partial \Delta=J F_{z}(\Delta) \text {. }
$$

Hence, the functions $K_{\mu \mu}(q)$ of (2.9) can be determined if the ground-state energy is known.

For even $N$, the ground-state of (2.1) has quantum number $S_{z}^{\mathrm{T}}=0$ and is nondegenerate. This has been proved rigorously for $0 \leq \Delta \leq 1, s=\frac{1}{2}$ (des Cloizeaux and Gaudin 1966), for $\Delta=1$, $s$ arbitrary (Lieb and Mattis 1962), and for $\Delta=0, s$ arbitrary (Mattis 1979). In Sec. 4 we shall assume that this statement is also true for $0<\Delta<1, s$ arbitrary, which is extremely plausible. Hence, at $T=0$ the dynamic structure factor $(2.3)$ can be written in the simple form

$$
S_{\mu \mu}(q, \omega)=\sum_{\lambda} M_{\lambda}^{\mu} \delta\left(\omega+E_{\mathrm{G}}-E_{\lambda}\right), \quad M_{\lambda}^{\mu}=2 \pi\left|\left\langle\mathrm{G}\left|S^{\mu}(q)\right| \lambda\right\rangle\right|^{2}
$$

where $|\mathrm{G}\rangle$ is the singlet ground state and $\lambda$ runs over all eigenstates of (2.1). 


\section{The planar $s=\frac{1}{2}$ antiferromagnet}

\subsection{The excitations dominating $S_{z z}(q, \omega)$}

We calculate dynamical correlation functions for finite systems by diagonalising $\mathcal{H}$ numerically and evaluating (2.11) directly with the exact eigenstates of $\mathcal{H}$. A convenient way of representing the result is to plot the energies and wavenumbers of the excited states $|\lambda\rangle$ contributing to (2.11) in a $(\omega, q)$ diagram. Figure 1 shows such a plot of $S_{z z}(q, \omega)$ for various $\Delta$ and $N=10$. The ground state is located at $\omega=0, q=0$. The matrix elements $M_{\lambda}^{z}$ are either given by their values or characterised by the choice of the symbol for the corresponding excited state, as described in the figure caption. The dominant contributions to $S_{z z}(q, \omega)$ evidently come from excitations lying between sinusoidal dispersion branches $\epsilon_{1}(q)$ and $\epsilon_{2}(q)$. In the thermodynamic limit $(N \rightarrow \infty)$ these states (shown as full circles) form a two-parameter continuum in $(q, \omega)$ space (called the spin-wave continuum A, SWC A) with boundaries $\epsilon_{1}(q)$ and $\epsilon_{2}(q)$. There are also states above $\epsilon_{2}(q)$ (open symbols) contributing to $S_{z z}(q, \omega)$. However, their spectral weight is observed to be lower by at least two orders of magnitude compared with SWC A states. In the XY limit $(\Delta=0)$ the contributions of such non-SWC states vanish altogether. Hence, for the following we will neglect these higher-lying excitations for all $\Delta$ and concentrate on the SWC A contributions to $S_{z z}(q, \omega)$.
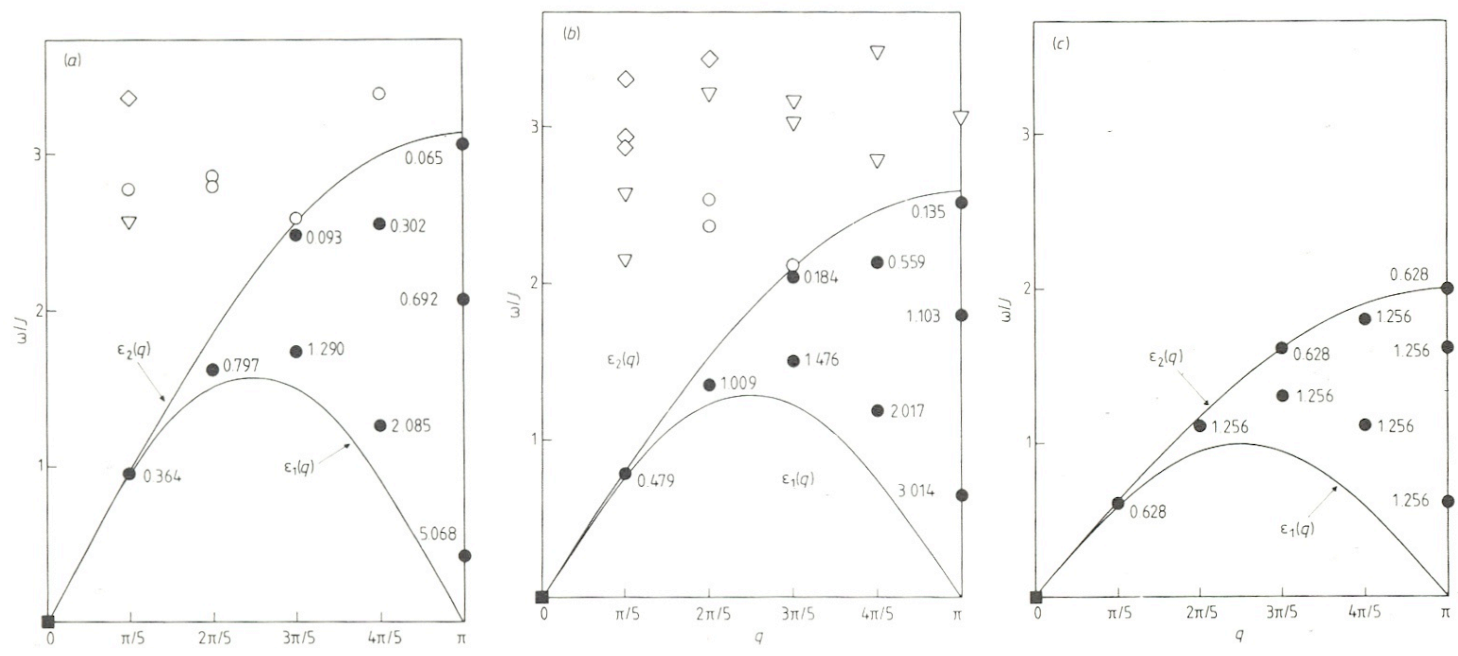

Figure 1. Spectral representation of $S_{z z}(q, \omega)$ at $T=0$ for the system (2.1) with $N=10$ and (a) $\Delta=1$, (b) $\Delta=0.5$, (c) $\Delta=0$. For each value of the wavenumber $q=(2 \pi / N) n, n=0,1, . . ., 5$, $S_{z z}(q, \omega)$ is a set of $\delta$-functions according to (2.11). The full square denotes the ground state and the positions of the other symbols determine energies and wavenumbers of the excitations contributing to $S_{z z}(q, \omega)$. Their spectral weights $M_{\lambda}^{z}$ are either represented by their value (for the full symbols) or characterised by the type of the symbol (for the open symbols) as follows: $M_{\lambda}^{z} \sim 10^{-3}$ (circle), $M_{\lambda}^{z} \sim 10^{-4}$ (triangle), $M_{\lambda}^{z} \sim 10^{-5}$ (diamond). The full symbols distinguish those states which can be identified as belonging to the 'class $\mathrm{C}$ ' in the Bethe ansatz description. In the thermodynamical limit they form a continuum (SWC A) between the two branches $\epsilon_{1}(q)$ and $\epsilon_{2}(q)$ drawn as full lines.

We can identify each SWC A excitation of figure 1with a member of the special 'class C' of eigenstates in the Bethe ansatz prescription (Griffiths 1964). Details of this identification, which is similar for all $\Delta$, are extensively described in our earlier work on the case $\Delta=1$ (Müller et al 1980, 1981b). With the method of des Cloizeaux and Gaudin (1966) we can calculate the SWC A excitation energies in the thermodynamic limit, yielding

$$
\omega_{\mathrm{m}}(q)=\frac{\pi J \sin \vartheta}{\vartheta} \sin \frac{q}{2} \cos \left(\frac{q}{2}-\frac{q_{\mathrm{m}}}{2}\right)
$$


with $\Delta=\cos \vartheta(0 \leq \vartheta \leq \pi / 2)$. Here $q$ denotes the wavenumber relative to that of the ground state, and $q_{\mathrm{m}}\left(0 \leq q_{\mathrm{m}} \leq q\right)$ labels the different branches in SWC A. In particular, $q_{\mathrm{m}}=0$ and $q_{\mathrm{m}}=q$ in (3.1) characterise the lower and the upper boundaries, respectively:

$$
\epsilon_{1}(q)=\frac{\pi J \sin \vartheta}{2 \vartheta} \sin q, \quad \epsilon_{2}(q)=\frac{\pi J \sin \vartheta}{\vartheta} \sin \frac{q}{2} .
$$

The density of states in the SWC A is found to be

$$
D(q, \omega) \equiv \frac{N}{4 \pi}\left(\frac{\partial \omega_{\mathrm{m}}(q)}{\partial q_{\mathrm{m}}}\right)^{-1}=\frac{N}{2 \pi}\left[\epsilon_{2}^{2}(q)-\omega^{2}\right]^{-1 / 2}, \quad \epsilon_{1}(q) \leq \omega \leq \epsilon_{2}(q) .
$$

Due to the fact that the ground state is non-degenerate and that any SWC A state is unambiguously identified by its location in $(q, \omega)$ space, we can write the SWC A contribution to $S_{z z}(q, \omega)$ as a product

$$
S_{z z}(q, \omega)=M_{\mathrm{A}}(q, \omega) D(q, \omega),
$$

where $M_{\mathrm{A}}(q, \omega)$ represents the squared matrix element $\left|\left\langle\mathrm{G}\left|S^{z}(q)\right| \mathrm{SWC} \mathrm{A}\right\rangle\right|^{2}$ between the ground state and the corresponding SWC A excitation with energy $\omega$.

It is well known that the $1 \mathrm{D}$ quantum spin system, which can be mapped onto the (2D classical) eight-vertex model (Baxter 1972, Johnson et al 1973), shows non-universal behaviour at its critical point $T=0$ with critical exponents depending on the interaction strengths, i.e. on $\Delta=J_{z} / J_{\perp}$ for the model system (2.1) (Luther and Peschel 1975). Hence, as a generalisation of the result

$$
M_{\mathrm{A}}(q, \omega)=\operatorname{const}\left(\frac{\epsilon_{2}^{2}(q)-\omega^{2}}{\omega^{2}-\epsilon_{1}^{2}(q)}\right)^{1 / 2}, \quad \Delta=1
$$

which we used for the isotropic Heisenberg AF (Müller et al 1980, 1981b) and the exact result

$$
M_{\mathrm{A}}(q, \omega)=\text { const }, \quad \Delta=0
$$

for the $X Y$ AF (Niemeijer 1967, Katsura et al 1970), we postulate for the squared matrix elements of the planar $s=\frac{1}{2} \mathrm{AF}(0 \leq \Delta \leq 1)$ the analytic expression

$$
M_{\mathrm{A}}(q, \omega)=\operatorname{const}\left(\frac{\epsilon_{2}^{2}(q)-\omega^{2}}{\omega^{2}-\epsilon_{1}^{2}(q)}\right)^{\alpha}
$$

with an exponent $\alpha$ whose explicit $\Delta$ dependence

$$
\alpha(\vartheta)=\frac{\pi / 2-\vartheta}{\pi-\vartheta}, \quad \cos \vartheta=\Delta
$$

will be justified below. Clearly, $\alpha=0$ in the $X Y$ limit and $\alpha=\frac{1}{2}$ in the Heisenberg limit. Equations (3.3), (3.4) and (3.7) now yield the following analytic expression for the OP dynamic structure factor:

$$
S_{z z}(q, \omega)=\frac{2 A}{B(1-\alpha, 1 / 2+\alpha)} \frac{\theta\left(\omega-\epsilon_{1}(q)\right) \theta\left(\epsilon_{2}(q)-\omega\right)}{\left[\omega^{2}-\epsilon_{1}^{2}(q)\right]^{\alpha}\left[\epsilon_{2}^{2}(q)-\omega^{2}\right]^{1 / 2-\alpha}} .
$$

$A$ is a prefactor to be determined and the beta function is a convenient normalising factor. In the $X Y$ limit (3.9) is exact with $A=2$ and $\alpha=0$ (Katsura et al 1970). For values of $q$ close to $\pi$ we find $\epsilon_{1} \simeq c(\pi-q)$ and $\epsilon_{2} \simeq 2 c$ with $c=\pi J \sin \vartheta /(2 \vartheta)$ and thus

$$
S_{z z}(q, \omega) \propto \theta(\omega-c(\pi-q))\left[\omega^{2}-c^{2}(\pi-q)^{2}\right]^{\alpha} .
$$

This is precisely the form given by Luther and Peschel (1975). These authors then use an exact relation between the spin chain (2.1) and the Baxter model in order to show that their exponent $\alpha$ is given by (3.8). Our hypothesis thus consists in extending a result which is exact for $q \simeq \pi$ to 
the full Brillouin zone. A severe test of our conjecture (3.9) for $S_{z z}(q, \omega)$, i.e. for our postulation of the analytic expression (3.7) for the squared matrix elements is based on the sum rule (2.8). The first frequency moment of (3.9) yields

$$
K_{z z}(q)=\frac{A J \sin \vartheta}{4 \vartheta}(1-\cos q) .
$$

It exactly reproduces the $q$-dependence of (2.9b) irrespective of $\Delta$, justifying therefore our ansatz for the dominating SWC A contributions to $S_{z z}(q, \omega)$. The prefactor $A$ of (3.9) is determined by equating (2.9b) and (3.10), yielding

$$
A(\vartheta)=-\frac{8 \vartheta}{\sin \vartheta} F_{x}(\vartheta)
$$

$F_{x}(\vartheta)$ is determined by the exact result for the ground-state energy of the $s=\frac{1}{2}$ system (Yang and Yang 1966)

$$
\epsilon_{\mathrm{G}}(\vartheta) / J=\frac{1}{4} \cos \vartheta-\frac{1}{2} \sin ^{2} \vartheta \int_{-\infty}^{+\infty} \frac{\mathrm{d} x}{\cosh (\pi x)(\cosh (2 \vartheta x)-\cos \vartheta)}
$$

and the relations (2.10). $A(\vartheta)$ is shown in figure 2. In particular, for the $X Y$ AF we have $F_{x}=$ $F_{y}=-1 / 2 \pi, F_{z}=-1 / \pi^{2}$, and for the Heisenberg AF $F_{x}=F_{y}=F_{z}=-\frac{1}{3}\left(\ln 2-\frac{1}{4}\right)$.

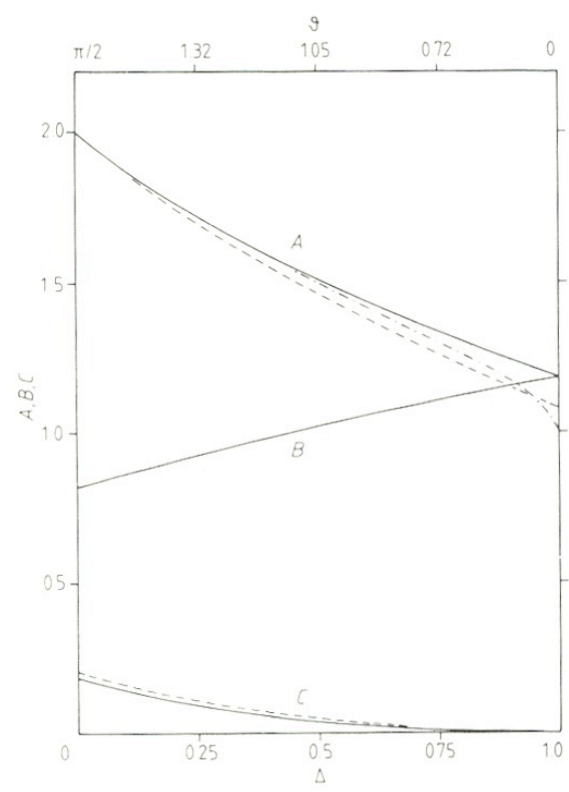

Figure 2. Anisotropy dependence of the prefactors $A, B$, and $C$ in the analytic expressions (3.9) and (3.18) for $S_{z z}(q, \omega)$ and $S_{x x}(q, \omega)$, respectively. The full lines represent $A, B, C$ as determined by the sum rule (2.8), the broken lines represent $A$ and $C$ as determined by finitechain extrapolations of integrated intensities, and the chain line represents $A$ as determined from exactly known susceptibility results. Details are explained in the text.

\subsection{The excitations dominating $S_{x x}(q, \omega)$}

As in Sec. 3.1. for the OP fluctuations we use finite-chain calculations in order to select those classes of excitations which dominate the IP dynamics. Figures 3(a), (b) show spectral representations of $S_{x x}(q, \omega)$ at $T=0$ for a ring of $N=10$ spins. The anisotropies are the same as in 
figures 1(b), (c). The case $\Delta=1$, where we have $S_{x x} \equiv S_{z z}$ was already shown in figure 1(a). It is evident from these figures that for $\Delta<1$ the excitation spectrum of $S_{x x}(q, \omega)$ is considerably more complicated than that of $S_{z z}(q, \omega)$. Nevertheless, we can select two classes of excitations which give the dominant contributions of $S_{x x}(q, \omega)$. One class, characterised by full circles, is again located between the two dispersion branches $\epsilon_{1}(q)$ and $\epsilon_{2}(q)$ of (3.2). In the isotropic limit $\Delta=1$ they are identified as belonging to the same triplet $\left(S^{\mathrm{T}}=1, S_{z}^{\mathrm{T}}=0, \pm 1\right)$ continuum as the SWC A states which dominate $S_{z z}(q, \omega)$. Hence, they form in the thermodynamic limit a two-parameter continuum with energies (3.1) and a density of states (3.3). We call it SWC B. Note that the (degenerate) triplet states $\left(S^{\mathrm{T}}=1\right)$ belonging either to SWC A $\left(S_{z}^{\mathrm{T}}=0\right)$ or SWC B $\left(S_{z}^{\mathrm{T}}= \pm 1\right)$ split for finite $N$ at $\Delta<1$, but remain degenerate in the thermodynamic limit. according to the work by des Cloizeaux and Gaudin (1966). The second class of excitations important for $S_{x x}(q, \omega)$ is characterised by full triangles in figure 3(a), (b). The contributions of these states are largest in the $X Y$ limit and vanish altogether in the Heisenberg limit. These excitations are identified as belonging for $\Delta=1$ to the class of unbound states with $S^{\mathrm{T}}=2$. In a preceding paper (Müller et al $1981 \mathrm{~b}$ ) we have proved that in the thermodynamic limit they form a two-parameter continuum (called SWC C) of the same shape as SWC A. This holds also for the corresponding triangle states in the case $\Delta<1$. In the limit $\Delta=1$ the SWC C states are excluded from contributing to $S_{x x}(q, \omega)$ by a selection rule involving the quantum number $S^{\mathrm{T}}$ (see e.g. Müller et al 1981a, b).
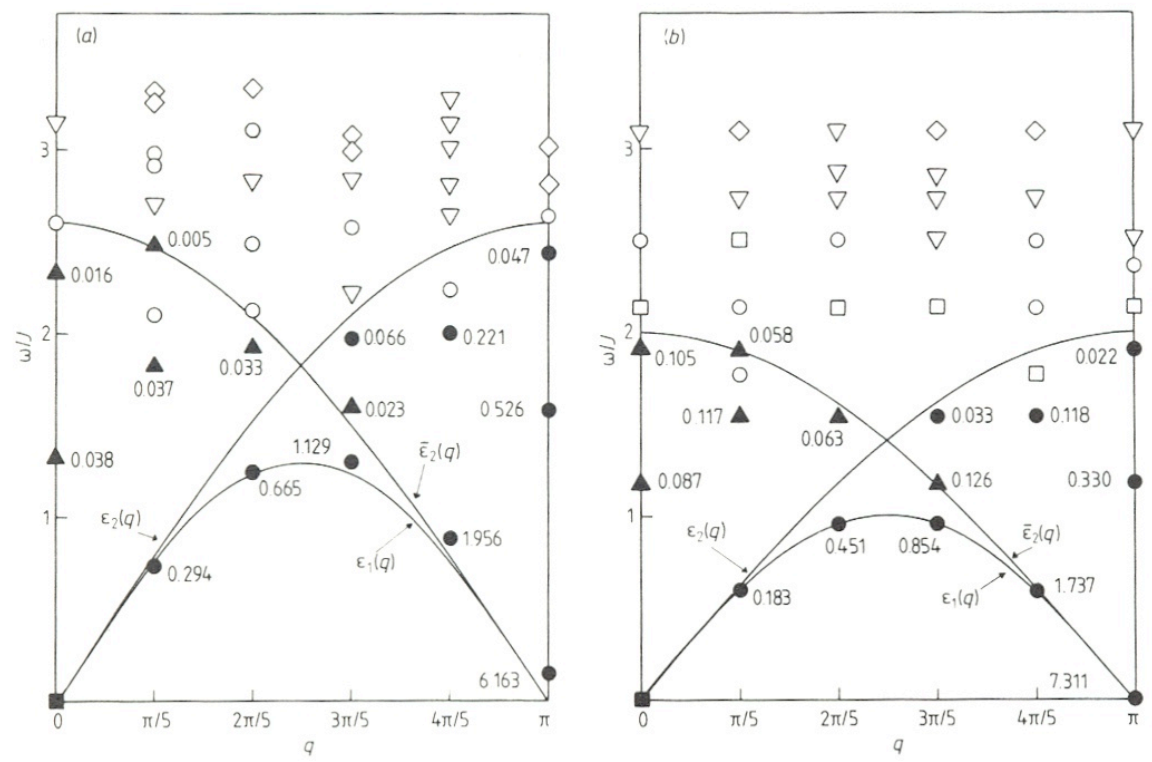

Figure 3. Spectral representation of $S_{x x}(q, \omega)$ at $T=0$ for the system (2.1) with $N=10$ and (a) $\Delta=0.5$, (b) $\Delta=0$. the case $\Delta=1$ is the same as shown in figure $1(\mathrm{c})$. The full square denotes the ground state and the other symbols determine energies and wavenumbers of the excitations contributing to $S_{x x}(q, \omega)$. Their spectral weights $M_{\lambda}^{x}$ are either represented by their value (for the full symbols) or characterised by the type of the symbol (for the open symbols) as follows: $M_{\lambda}^{x} \sim 10^{-2}$ (square), $M_{\lambda}^{x} \sim 10^{-3}$ (circle), $M_{\lambda}^{x} \sim 10^{-4}$ (triangle), $M_{\lambda}^{x} \sim 10^{-5}$ (diamond). The full circles and the full triangles, which are identified as belonging to the 'class $\mathrm{C}^{\prime}$ in the Bethe ansatz description, form - in the thermodynamic limit - the two continua SWC Band SWC C with boundaries $\epsilon_{1}(q), \epsilon_{2}(q)$ and $\bar{\epsilon}_{1}(q)$ and $\bar{\epsilon}_{2}(q)$, respectively. In order to emphasise the essential features in the figure, we have corrected for a finite-size effect by subtracting from the finite-chain excitation energies a constant amount $\epsilon_{0}=0.12 J$ for $\Delta=0.5$ and $\epsilon_{0}=0.15 J$ for $\Delta=0$..

For reasons which are extensively discussed in the latter reference, the SWC C states appear in $S_{x x}(q, \omega)$ with wavenumbers $q$ replaced by $\bar{q} \equiv \pi-q$. Thus the lower and upper boundaries of 
SWC C are given by

$$
\bar{\epsilon}_{1}(q)=\epsilon_{1}(q)=\frac{\pi J \sin \vartheta}{2 \vartheta} \sin q, \quad \bar{\epsilon}_{2}(q)=\frac{\pi J \sin \vartheta}{\vartheta} \cos \frac{q}{2}
$$

and the density of states by

$$
\bar{D}(q, \omega)=\frac{N}{2 \pi}\left[\bar{\epsilon}_{2}^{2}(q)-\omega^{2}\right]^{-1 / 2}, \quad \bar{\epsilon}_{1}(q) \leq \omega \leq \bar{\epsilon}_{2}(q) .
$$

There are excitations contributing to $S_{x x}(q, \omega)$ which do not belong to either SWC B or SWC C. They are shown as open symbols in figure 3(a), (b) (and in figure 1(a) for $\Delta=1$ ). Their contributions are rather small except close to the $X Y$ limit, where some of the non-SWC matrix elements seem no longer to be negligible. Nevertheless we shall consider in the following only the SWC excitations for the $T=0$ dynamics. In analogy to our procedure in Sec. 3.1 we can write for their contributions to the IP structure factor the generalised ansatz

$$
S_{x x}(q, \omega)=M_{\mathrm{B}}(q, \omega) D(q, \omega)+M_{\mathrm{C}}(q, \omega) \bar{D}(q, \omega)
$$

where $M_{\mathrm{B}}(q, \omega)$ and $M_{\mathrm{C}}(q, \omega)$ represent the squared matrix elements $\left|\left\langle\mathrm{G}\left|S^{x}(q)\right| \mathrm{SWC} \mathrm{B}\right\rangle\right|^{2}$ and $\left|\left\langle\mathrm{G}\left|S^{x}(q)\right| \mathrm{SWC} \mathrm{C}\right\rangle\right|^{2}$, respectively. We postulate that these two quantities can be expressed by the same type of analytic form as (3.7)

$$
M_{\mathrm{B}}(q, \omega)=\operatorname{const}\left(\frac{\epsilon_{2}^{2}(q)-\omega^{2}}{\omega^{2}-\epsilon_{1}^{2}(q)}\right)^{\beta}, \quad M_{\mathrm{C}}(q, \omega)=\operatorname{const}\left(\frac{\bar{\epsilon}_{2}^{2}(q)-\omega^{2}}{\omega^{2}-\bar{\epsilon}_{1}^{2}(q)}\right)^{\gamma} .
$$

Again, the anisotropy dependence of the exponents is related to exactly known exponents of the Baxter model. The results which are found in the work by Luther and Peschel (1975) read

$$
\beta(\vartheta)=\frac{1}{2}+\frac{\vartheta}{2 \pi}, \quad \gamma(\vartheta)=\frac{1}{2} \frac{(\vartheta / \pi)^{2}}{\vartheta / \pi-1} .
$$

In the Heisenberg limit we have $\beta=\frac{1}{2}, \gamma=0$ and in the $X Y$ limit $\beta=\frac{3}{4}, \gamma=-\frac{1}{4}$. With (3.15), (3.14) and (3.16) we end up with the following expression for the IP structure factor:

$$
\begin{aligned}
S_{x x}(q, \omega) & =S_{x x}^{\mathrm{B}}(q, \omega)+S_{x x}^{\mathrm{C}}(q, \omega) \\
S_{x x}^{\mathrm{B}}(q, \omega) & =\frac{2 B}{B(1-\beta, 1 / 2+\beta)} \frac{\theta\left(\omega-\epsilon_{1}(q)\right) \theta\left(\epsilon_{2}(q)-\omega\right)}{\left[\omega^{2}-\epsilon_{1}^{2}(q)\right]^{\beta}\left[\epsilon_{2}^{2}(q)-\omega^{2}\right]^{1 / 2-b e t a}} \\
S_{x x}^{\mathrm{C}}(q, \omega) & =\frac{2 C}{B(1-\gamma, 1 / 2+\gamma)} \frac{\theta\left(\omega-\bar{\epsilon}_{1}(q)\right) \theta\left(\bar{\epsilon}_{2}(q)-\omega\right)}{\left[\omega^{2}-\bar{\epsilon}_{1}^{2}(q)\right]^{\gamma}\left[\bar{\epsilon}_{2}^{2}(q)-\omega^{2}\right]^{1 / 2-\gamma}} .
\end{aligned}
$$

The prefactors $B$ and $C$ are determined below, and we use again the beta function as convenient normalising factor. An important check for the validity of our analytic expression (3.18) is again the sum rule (2.8). The first frequency moment of (3.18) is evaluated as

$$
K_{x x}(q)=\frac{B J \sin \vartheta}{4 \vartheta}(1-\cos q)+\frac{C J \sin \vartheta}{4 \vartheta}(1+\cos q) .
$$

It is indeed consistent with the exact result (2.9a), provided $B$ and $C$ have the following $\vartheta$ dependence:

$$
\begin{aligned}
& B(\vartheta)=-2 \vartheta \cot \frac{\vartheta}{2}\left[F_{x}(\vartheta)+F_{z}(\vartheta)\right], \\
& C(\vartheta)=-2 \vartheta \tan \frac{\vartheta}{2}\left[F_{x}(\vartheta)-F_{z}(\vartheta)\right],
\end{aligned}
$$

where the NN correlation functions are determined by (2.10) and (3.12). The anisotropy dependence of $B$ and $C$ as given by (3.20) is shown in figure 2 as full lines. Clearly, in the isotropic limit $(\vartheta=0)$ we have $B=A$ and $C=0$. Hence, all ingredients of our conjectures (3.9) and (3.18) are determined. In the following we shall discuss their implications for various observables, and estimate the accuracy of the approach by comparing the predictions with exact results wherever they are available. 


\subsection{Lineshapes of $S_{z z}(q, \omega)$ and $S_{x x}(q, \omega)$}

Figure 4 shows $S_{z z}(q, \omega)$ at fixed $q=4 \pi / 5$ as a function of $\omega$ for various anisotropies. In the $X Y$ limit $(\Delta=0, \alpha=0) M_{\mathrm{A}}(q, \omega)$ is a constant and $S_{z z}(q, \omega)$ reflects the SWC A density of states with a square-root divergence at the upper boundary $\epsilon_{2}(q)$ and a finite step at $\epsilon_{1}(q)$. For intermediate anisotropies $(0<\Delta<1,0<\alpha<1 / 2) M_{\mathrm{A}}(q, \omega)$ is no longer a constant, but diverges at $\epsilon_{1}(q)$ and vanishes at $\epsilon_{2}(q)$. This leads to a weaker singularity in $S_{z z}(q, \omega)$ at $\epsilon_{2}(q)$ and gives rise to a second divergence at the lower boundary $\epsilon_{1}(q)$. For $\Delta=\frac{1}{2}, \alpha=\frac{1}{4}$ the two singularities are equally strong which gives rise to crossover phenomena as described below. In the Heisenberg limit $\left(\Delta=1, \alpha=\frac{1}{2}\right)$ the matrix elements (3.7) vanish sufficiently rapidly at $\epsilon_{2}(q)$ in order to overcome the density of states singularity. On the other hand the divergence at $\epsilon_{1}(q)$ has become quite strong so that most of the spectral weight is concentrated near that lower boundary. From inelastic neutron scattering experiments on corresponding quasi-1D planars $=\frac{1}{2} \mathrm{AF}$ compounds, we therefore expect that they will reveal at low $T$ a two-peak structure according to equation (3.9). Of course, for $\Delta$ either close to the Heisenberg limit or close to the $X Y$ limit, one or the other of the divergences in $S_{z z}(q, \omega)$ may be too weak to produce an observable structure in the scattering cross section. Moreover, for $q<\pi / 2$ where the SWC A is rather narrow, the two peaks may no longer be resolved as independent structures.

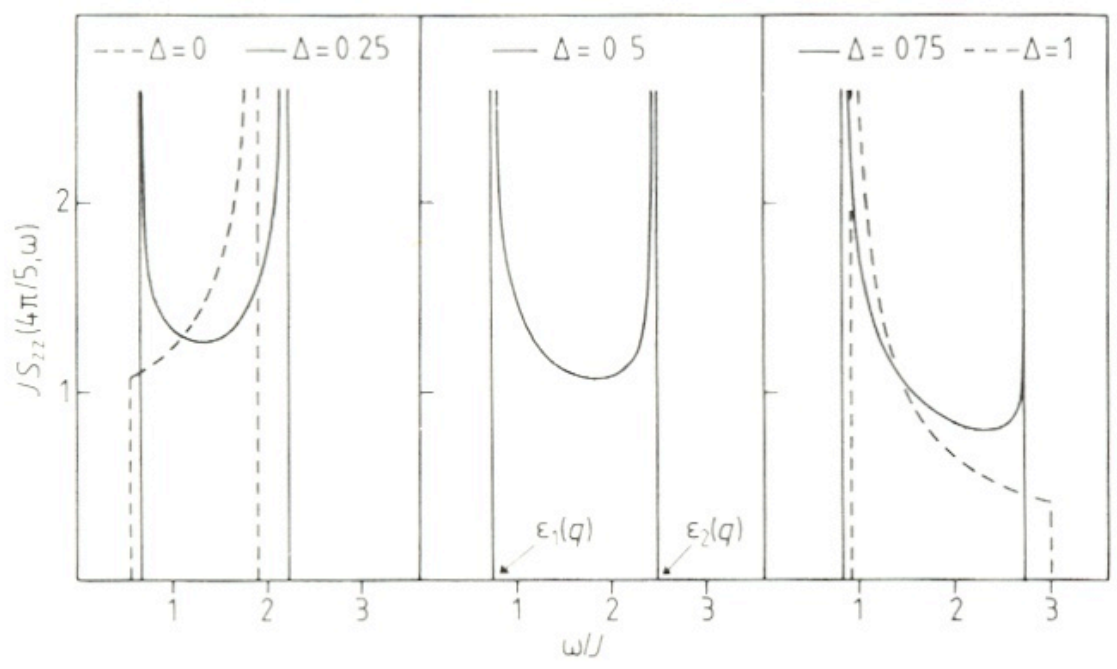

Figure 4. Out-of-plane dynamic structure factor $S_{z z}(q, \omega)$ of equation (3.9) versus $\omega$ at fixed $q=4 \pi / 5$ for various anisotropies.

Figures 5(a), (b) show $S_{x x}(q, \omega)$ as a function of $\omega$ for various $\Delta$ at fixed $q=4 \pi / 5$ and $q=\pi / 5$, respectively. Let us first consider figure 5 (a) for a wavenumber close to the zone boundary. Here $S_{x x}^{\mathrm{B}}(q, \omega)$ is dominant for all $\Delta$, having a divergence at $\epsilon_{1}(q)$ and a tail out to $\epsilon_{2}(q)$. With $\Delta$ decreasing from unity, the divergence at $\epsilon_{1}(q)$ becomes stronger and the tail less pronounced. The contribution of $S_{x x}^{\mathrm{C}}(q, \omega)$ to the intensity pattern is a narrow peak at $\bar{\epsilon}_{2}(q)$ (shown as a broken line) with a very short tail down to $\epsilon_{1}(q)$. It is located where $S_{x x}^{\mathrm{B}}$ has large spectral weight. The integrated intensity of $S_{x x}^{\mathrm{C}}$ at $q=4 \pi / 5$ reaches at most $4 \%$ of the $S_{x x}^{\mathrm{B}}$ integrated intensity in the $X Y$ limit and vanishes in the isotropic limit. Therefore, what will be observable in inelastic neutron scattering experiments is essentially the $S_{x x}^{\mathrm{B}}$ peak at $\epsilon_{1}(q)$ with a lineshape asymmetry which is most pronounced for $\Delta=1$.

The situation is quite different for $q=\pi / 5$, a wavenumber close to the zone centre. Here, the contributions to $S_{x x}^{\mathrm{C}}(q, \omega)$ from SWC B and SWC C are located at different energies. $S_{x x}^{\mathrm{B}}(q, \omega)$ has a narrow peak at $\epsilon_{1}(q)$ with short tail up to $\epsilon_{2}(q)$ shown with full lines in figure $5(\mathrm{~b})$. On the other hand, the spectral weight of $S_{x x}^{\mathrm{C}}$ (shown by broken lines in figure $5(\mathrm{~b})$ ) is distributed over a wider 
range of frequencies between $\epsilon_{1}(q)$ and $\bar{\epsilon}_{2}(q)$. It has a divergence at $\bar{\epsilon}_{2}(q)$ and a weak tail down to $\epsilon_{1}(q)$. At this wavenumber $(q=\pi / 5)$, the integrated intensity of $S_{x x}^{\mathrm{C}}$ reaches about $40 \%$ (for $\Delta=0$ ) of the $S_{x x}^{\mathrm{B}}$ integrated intensity. It decreases to $10 \%$ for $\Delta=0.5$ and vanishes for $\Delta=0$. Hence, for small $\Delta$ we expect that in inelastic neutron scattering both peaks should be observable. As $q$ increases, the $S_{x x}^{\mathrm{B}}$ peak becomes broader and gains intensity whereas $S_{x x}^{\mathrm{C}}$ loses intensity and may no longer be detectable.
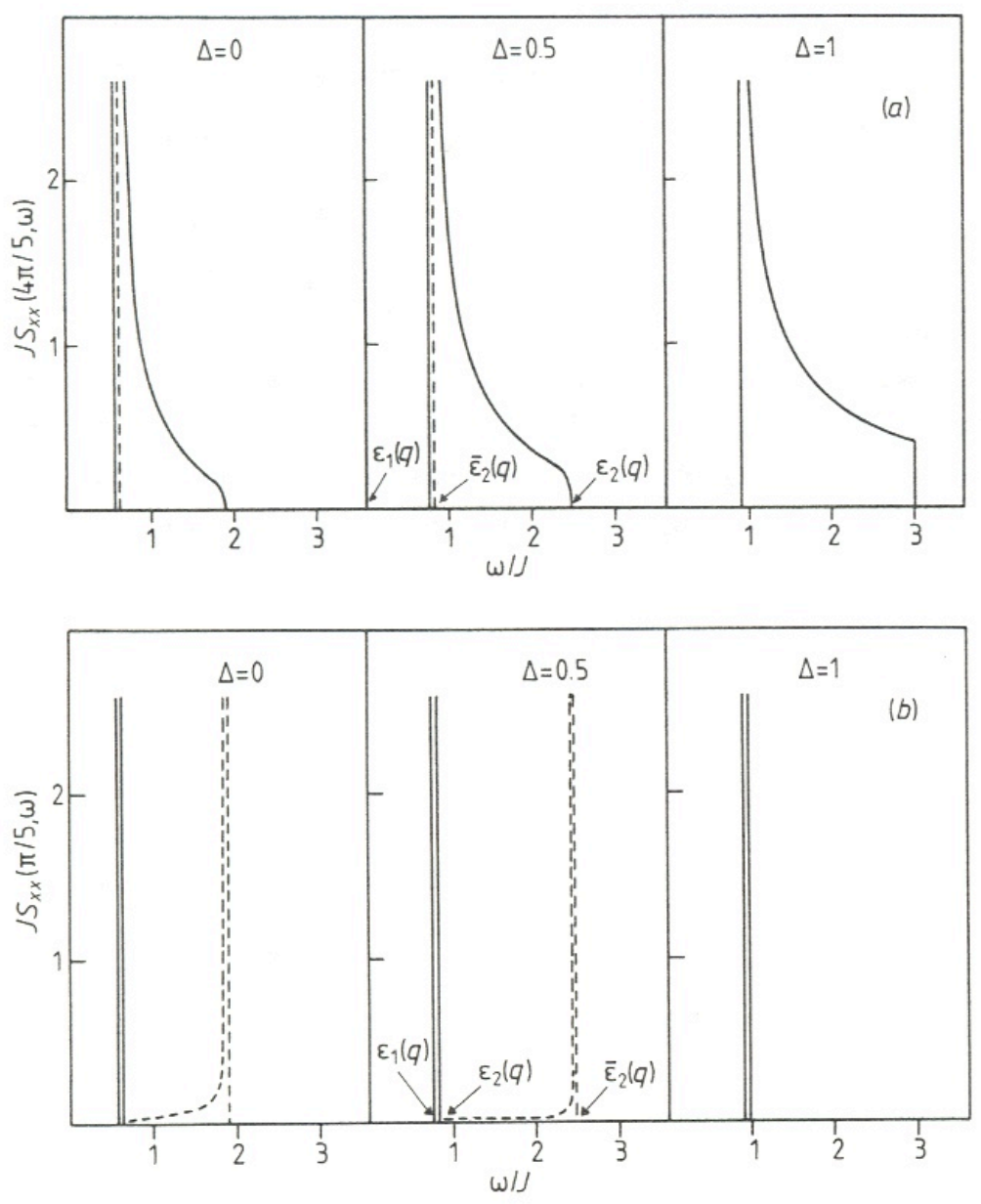

Figure 5. In-plane dynamic structure factor $S_{z z}(q, \omega)$ of equation (3.18) versus $\omega$ at fixed wavenumber: (a) $q=4 \pi / 5$, (b) $q=\pi / 5$, for various anisotropies $\Delta=J_{z} / J_{\perp}$. The full (broken) lines represent the contributions from SWC B (SWC C).

\subsection{Static correlation functions}

The integrated intensity (2.4) of the OP fluctuations (3.9) is found in terms of a hypergeometric function (Gradshteyn and Ryzhik 1965)

$$
I_{z z}(q)=\frac{A}{2 \pi} \tan \frac{q}{2}{ }_{2} F_{1}\left(\frac{1}{2}, 1-\alpha ; \frac{3}{2} ;-\tan ^{2} \frac{q}{2}\right) .
$$


It is shown in figure 6 for $\Delta=0,0.5,1.0$. For small $q$ it increases linearly, $I_{z z}(q)=(A / 4 \pi) q+\mathrm{O}\left(q^{2}\right)$, and at $q=\pi$ (i.e. $\bar{q}=0$ ) it stays finite with a cusp-like behaviour:

$$
I_{z z}(q)=\frac{A}{4 \sqrt{\pi}} \frac{\Gamma(1 / 2-\alpha)}{\Gamma(1-\alpha)}-\frac{A}{\pi} \frac{\bar{q}^{1-2 \alpha}}{2^{2(1-\alpha)}(1-2 \alpha)}
$$

except for $\Delta=1\left(\alpha=\frac{1}{2}\right)$ where $I_{z z}(q)$ has a logarithmic divergence for $q=\pi$. In the $X Y$ limit the linear term is already the exact result for all $q$. In the Heisenberg limit the hypergeometric function reduces to

$$
I_{z z}(q)=\frac{A}{2 \pi} \ln \frac{1+\sin (q / 2)}{\cos (q / 2)} .
$$

The behaviour near $q=\pi$ (cusp or divergence) determines the asymptotic behaviour of the correlations in real space:

$$
\left\langle S_{l}^{z} S_{l+R}^{z}\right\rangle \sim p_{z} \frac{(-1)^{R}}{R^{2(1-\alpha)}}, \quad p_{z}=\frac{A \cos (\pi-\pi \alpha) \Gamma(2-2 \alpha)}{\pi^{2} 2^{2-2 \alpha}(2 \alpha-1)} .
$$

We observe that the OP correlations are strongest in the isotropic limit $\left(\alpha=\frac{1}{2}\right)$. In the opposite limit $\Delta=0, \alpha=0)$ the uniformly decaying term is of the same order as the oscillating term yielding (Lieb et al 1961) $\left\langle S_{l}^{z} S_{l+R}^{z}\right\rangle=\left(2 \pi^{2}\right)^{-1}\left[(-1)^{R}-1\right] / R^{2}$.

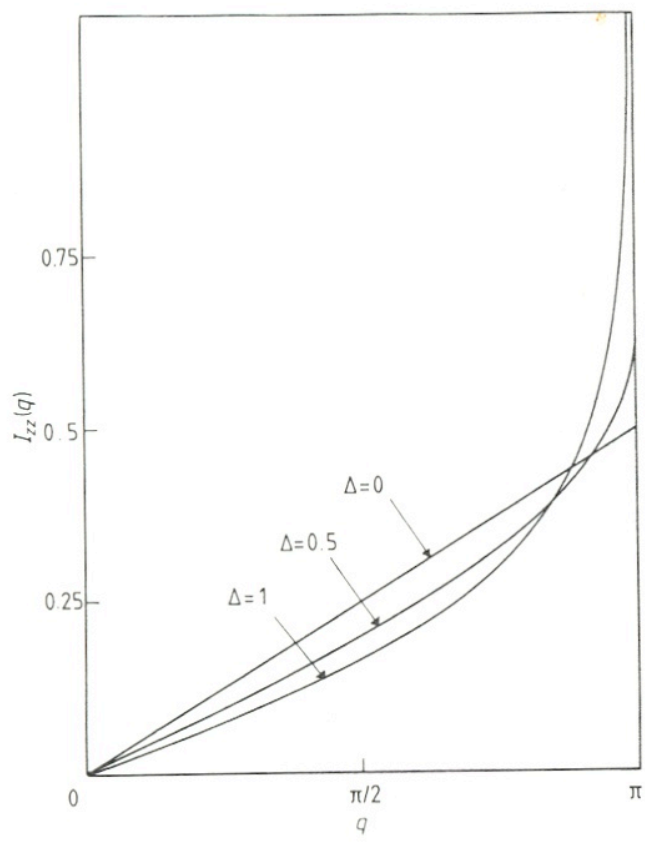

Figure 6. Out-of-plane integrated intensity $I_{z z}(q)$ of equation $(3.21)$ for $\Delta=1.0,0.5,0$. Details are explained in the text.

The IP integrated intensity of (3.18) is calculated as

$$
\begin{aligned}
I_{x x}(q) & =\frac{B}{2 \pi} \tan \frac{q}{2}{ }_{2} F_{1}\left(\frac{1}{2}, 1-\beta ; \frac{3}{2} ;-\tan ^{2} \frac{q}{2}\right) \\
& +\frac{C}{2 \pi} \cot \frac{q}{2}{ }_{2} F_{1}\left(\frac{1}{2}, 1-\gamma ; \frac{3}{2} ;-\cot ^{2} \frac{q}{2}\right)
\end{aligned}
$$

For $q \rightarrow 0$ it is nonzero, $I_{x x}(q)=(C / 4 \sqrt{\pi})\left[\Gamma\left(\frac{1}{2}-\gamma\right) / \Gamma(1-\gamma)\right]+\mathrm{O}(q)$ except for $\Delta=1$. At the zone boundary $I_{x x}(q)$ diverges with a power law $\left.I_{x x}(q) \sim \bar{q}^{1-2 \beta}(\bar{q} \ll 1)\right)$ for $\Delta<1$ and logarithmically 
for $\Delta=1$. Figure 7 shows $I_{x x}(q)$ for $\Delta=1$ and $\Delta=0$. SWC C contributes only for $\Delta<1$. The part of $I_{x x}(q)$ which is due to SWC C for $\Delta=0$ is shown by a broken line. The asymptotic behaviour of the IP correlations in real space is

$$
\left\langle S_{l}^{x} S_{l+R}^{x}\right\rangle \sim p_{x} \frac{(-1)^{R}}{R^{2(1-\beta)}} \quad p_{x}=\frac{B \cos (\pi-\pi \beta) \Gamma(2-2 \beta)}{\pi^{2} 2^{2-2 \beta}(2 \beta-1)} .
$$

Thus, with $\Delta \rightarrow 0\left(\beta \rightarrow \frac{3}{4}\right)$ the spin correlations in the easy plane are growing in range without, however, building up true long-range order (LRO). For the $X Y$ AF the prefactor of the leading term takes the value $p_{x}=\pi^{-5 / 2}(1+\pi / 2) \simeq 0.1470$. The deviation from the exact result $p_{x}=0.1471 \ldots$ obtained by Vaidya and Tracy (1978) is indeed minute, supporting the validity of our approach.

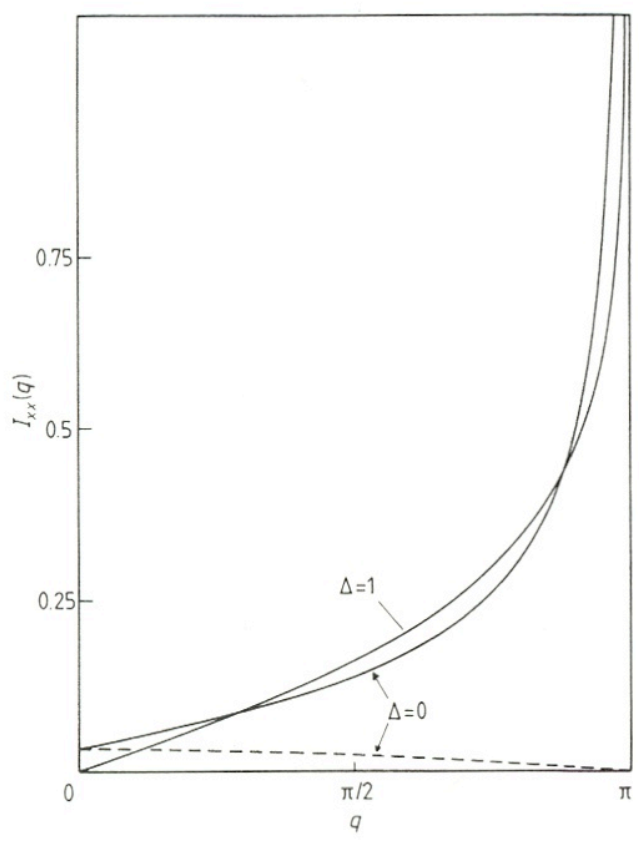

Figure 7. In-plane integrated intensity $I_{x x}(q)$ of equation (3.24) for $\Delta=0$ and $\Delta=1$ (full lines). The broken line represents the $S_{x x}^{C}$ part of (3.18) for $\Delta=0$. For $\Delta=1$ this part vanishes.

A somewhat less sensitive way to estimate the accuracy of our approach is provided by extrapolating finite-chain results of the integrated intensity for $N=2$ to 10 . We compare the extrapolated result for $I_{z z}(2 \pi / N) /(2 \pi / N)$ with the prefactor $A(\vartheta) / 4 \pi$ of the linear term in $q$ of equation (3.21) and determine $A$ such that they are in agreement. This $A(\vartheta)$ is shown as a broken line in figure 2 . Its deviation from (3.11) is zero for $\Delta=0$ and reaches about $8 \%$ for $\Delta=1$. The same has been done for the prefactor $C(\vartheta)$ by comparing the extrapolated $I_{x x}(0)$ with (3.24). The resulting $C$ is also included as a broken line in figure 2 and shows rather small deviation. Due to the interference of SWC B and SWC C in $I_{x x}(q)$ a convergent extrapolation which would allow for an estimate of $B(\vartheta)$ has not been possible. Yet for $\vartheta=\pi / 2$ we find that $B(\vartheta)$ as determined by (3.20) differs by less than $0.1 \%$ from $B(\vartheta)$ determined from $(3.25)$ with the exactly known $p_{x}$.

\subsection{Static susceptibility}

The OP component of the q-dependent susceptibility is calculated as (Gradshteyn and Ryzhik 1965)

$$
\chi_{z z}(q)=\frac{\vartheta A(\vartheta)}{\pi^{2} J \sin \vartheta}{ }_{2} F_{1}\left(1, \alpha+\frac{1}{2} ; \frac{3}{2} ; \sin ^{2} \frac{q}{2}\right) .
$$


In the Heisenberg and $X Y$ limits (3.26) reduces to the more elementary expressions

$$
\begin{gathered}
\chi_{z z}(q)=\frac{A}{\pi^{2} J} \frac{q}{\sin q} \quad(\Delta=1), \\
\chi_{z z}(q)=\frac{A}{4 \pi J \sin (q / 2)} \ln \frac{1+\sin (q / 2)}{1-\sin (q / 2)} \quad(\Delta=0) .
\end{gathered}
$$

At the zone boundary it diverges as a power law $\chi_{z z}(q) \sim \bar{q}^{-2 \alpha}$ for $\Delta>0$ and logarithmically for $\Delta=0$. At $q=0$ it stays finite $\left[{ }_{2} F_{1} \rightarrow 1\right.$ in (3.26)]. Yang and Yang (1966) have already obtained the exact result for the uniform $(q=0)$ susceptibility

$$
\chi_{z z}(0)=\frac{\vartheta}{J \pi(\pi-\vartheta) \sin \vartheta} .
$$

Together with (3.26) for $q=0$ this provides an independent way to determine $A(\vartheta)$. The result $A(\vartheta)=\pi /(\pi-\vartheta)$ is included in figure 2 as a chain line. The deviation from (3.11) is not more than a few percent, except close to $\Delta=1$, where it reaches $15 \%$. This agreement again demonstrates the consistency of our approach with exact results. The discrepancy near $\Delta=1$ is due to the neglect of higher-lying triplet excitations as is extensively discussed in a preceding paper (Müller et al 1981b). The IP susceptibility is evaluated as

$$
\chi_{x x}(q)=\frac{\vartheta B(\vartheta)}{\pi^{2} J \sin \vartheta}{ }_{2} F_{1}\left(1, \beta+\frac{1}{2} ; \frac{3}{2} ; \sin ^{2} \frac{q}{2}\right)+\frac{\vartheta C(\vartheta)}{\pi^{2} J \sin \vartheta}{ }_{2} F_{1}\left(1, \gamma+\frac{1}{2} ; \frac{3}{2} ; \sin ^{2} \frac{q}{2}\right)
$$

At $q=0$ it is finite for all $\Delta$,

$$
\chi_{x x}(0)=\frac{\vartheta B(\vartheta)}{\pi^{2} J \sin \vartheta}+\frac{\vartheta C(\vartheta)}{2 \pi^{2} J \sin \vartheta} \frac{\Gamma(-\gamma)}{\Gamma(1-\gamma)},
$$

and at the zone boundary it has a power-law divergence $\chi_{x x}(q) \sim \bar{q}^{-2 \beta}$. To the best of our knowledge there is no exact result available for $\chi_{x x}(0)$ at $\Delta<1$ which could provide a further check of the validity of our theory.

\subsection{Autocorrelation functions}

The autocorrelation functions $F_{\mu \mu}(\omega)$ are obtained by integrating (3.9) and (3.18) over the Brillouin zone. In the limits $\Delta=0$ and $\Delta=1 F_{z z}(\omega)$ can be evaluated as a combination of elliptic integrals. For $\Delta=1$ it is shown in figure 5 of the paper by Groen et al (1980). For low frequencies the OP autocorrelation function increases from zero with a power law as

$$
F_{z z}(\omega) \sim \omega^{1-2 \alpha}, \quad \omega \ll J \quad(0 \leq \Delta<1),
$$

whereas the IP component diverges as

$$
F_{x x}(\omega) \sim \omega^{1-2 \beta}, \quad \omega \ll J \quad(0 \leq \Delta<1) .
$$

In the isotropic limit $\Delta=1$ both functions stay finite for $\omega \rightarrow 0$ :

$$
F_{x x}(\omega)=F_{z z}(\omega)=\frac{A}{\pi}+\mathrm{O}(\omega) \quad(\Delta=1) .
$$

Thus, there is strong influence of the planar anisotropy on the low-frequency autocorrelation functions. This effect should clearly be observable in proton spin-lattice relaxation measurements on appropriate quasi-1D planar AF compounds.

Apart from frequency-dependent quantities time-dependent correlation functions $\left\langle S_{l}^{\mu}(t) S_{l+R}^{\mu}\right\rangle$, in particular the long-time asymptotic behaviour of the autocorrelation function $\left\langle S_{l}^{\mu}(t) S_{l}^{\mu}\right\rangle$, which 
is the Fourier transform of $F_{\mu \mu}(\omega)$, are of interest. Due to the absence of LRO, we expect a characteristic decay for $t \gg J^{-1}$ in analogy to the observed power-law decay of the static correlations $\left\langle S_{l}^{\mu} S_{l+R}^{\mu}\right\rangle$ for $R \gg 1$. In the case of the time tails, however, new remarkable features appear, not present in the statics. Clearly, the leading term in $\left\langle S_{l}^{\mu}(t) S_{l}^{\mu}\right\rangle$ for large time is related to the 'strongest' singularity in $F_{\mu \mu}(\omega)$. Starting from our results (3.9) and (3.18) it is evident that such van Hove singularities occur at the three frequencies

$$
\omega_{0}=0, \quad \omega_{1}=\epsilon_{1}\left(\frac{\pi}{2}\right)=\pi J \frac{\sin \vartheta}{2 \vartheta}, \quad \omega_{2}=\epsilon_{2}(\pi)=2 \omega_{1} .
$$

We find that the long-time behaviour of $\left\langle S_{l}^{z}(t) S_{l}^{z}\right\rangle$ is dominated by three terms in competition with each other:

$$
\left\langle S_{l}^{z}(t) S_{l}^{z}\right\rangle \sim a_{0} t^{-\alpha_{0}}+a_{1} t^{-\alpha_{1}} \mathrm{e}^{\mathrm{i} \omega_{1} t}+a_{2} t^{-\alpha_{2}} \mathrm{e}^{\mathrm{i} \omega_{2} t},
$$

where the exponents are related to $\alpha(\vartheta)$ of $(3.8)$ as

$$
\alpha_{0}=2(1-\alpha), \quad \alpha_{1}=\frac{3}{2}-\alpha, \quad \alpha_{2}=1+\alpha .
$$

For the $x x$ correlations, on the other hand, we obtain three dominant terms from both $S_{x x}^{\mathrm{B}}(q, \omega)$ and $S_{x x}^{\mathrm{C}}(q, \omega)$ of $(3.18)$.

$$
\left\langle S_{l}^{x}(t) S_{l}^{x}\right\rangle \sim \sum_{j=0}^{2}\left\{b_{j} t^{-\beta_{j}} \mathrm{e}^{\mathrm{i} \omega_{j} t}+c_{j} t^{-\gamma_{j}} \mathrm{e}^{\mathrm{i} \omega_{j} t}\right\}
$$

with exponents

$$
\begin{array}{lll}
\beta_{0}=2(1-\beta), & \beta_{1}=\frac{3}{2}-\beta, & \beta_{2}=1+\beta ; \\
\gamma_{0}=2(1-\gamma), & \gamma_{1}=\frac{3}{2}-\gamma, & \gamma_{2}=1+\gamma ;
\end{array}
$$

The constants $a_{j}, b_{j}, c_{j}$, which involve the amplitudes and phases of the competing terms, are all nonzero except for $\Delta=1$ where all $c_{j}$ vanish.

The $\Delta$-dependence of the exponents $\alpha_{j}, \beta_{j}, \gamma_{j}$ shown in figure 8 gives rise to dramatic features. For $\Delta=0$ the $z z$ autocorrelation function at $t \gg J^{-1}$ is dominated by an oscillating term $\left\langle S_{l}^{z}(t) S_{l}^{z}\right\rangle \sim \mathrm{e}^{\mathrm{i} \omega_{2} t} / t$. This is already implicit in the work of Katsura et al (1970) who give (for $\Delta=0)$ the exact result of $\left\langle S_{l}^{z}(t) S_{l}^{z}\right\rangle$ in terms of Bessel and Struve functions. There exist, however, no corresponding exact results for $\Delta>0$. Our approximate approach predicts - as is illustrated in figure $8(\mathrm{a})$ - that there is a crossover at $\Delta=0.5$ where a term $\mathrm{e}^{\mathrm{i} \omega_{1} t} t^{-\alpha_{1}}$ becomes dominant, i.e. a term oscillating with half the original frequency. This corresponds to the crossover of the strengths of the singularities of $S_{z z}(q, \omega)$ at $\epsilon_{1}(q)$ and $\epsilon_{2}(q)$ mentioned in Sec. 3.3. This term stays dominant up to $\Delta=1$ where a term with uniform decay also contributes to leading order: $\left\langle S_{l}^{z}(t) S_{l}^{z}\right\rangle \sim\left(a_{0}+a_{1} \mathrm{e}^{\mathrm{i} \omega_{2} t}\right) t^{-1}$. For $\Delta=1$ this result also holds for the $x x$ autocorrelation function. In this case, however, the first uniform term predominates for all $\Delta<1:\left\langle S_{l}^{x}(t) S_{l}^{x}\right\rangle \sim b_{0} t^{-2(1-\beta)}$, whereas the second uniform term $c_{0} t^{-2(1-\gamma)}$ decays even more rapidly than the ordinary $t^{-2}$ term linear in $\omega$ which is always present in $F_{\mu \mu}(\omega)$.

For the $X Y$ case we can compare our prediction with the exact result. The exact asymptotic behaviour of $\left\langle S_{l}^{x}(t) S_{l+R}^{x}\right\rangle$ as obtained by Vaidya and Tracy (1978) reads

$$
\left\langle S_{l}^{x}(t) S_{l+R}^{x}\right\rangle \sim \frac{1}{4} \mathrm{e}^{1 / 2} 2^{2 / 3} A^{-6}(-1)^{R}\left(R^{2}-J^{2} t^{2}\right)^{-1 / 4} .
$$

Here, $A=1.282427 \ldots$ is Glaisher's constant. The Fourier transform of (3.18), on the other hand, yields to leading order

$$
\left\langle S_{l}^{x}(t) S_{l+R}^{x}\right\rangle \sim \pi^{-5 / 2}(1+\pi / 2)(-1)^{R}\left(R^{2}-J^{2} t^{2}\right)^{-1 / 4} .
$$

Thus, it reproduces the correct $R$ and $t$ dependence. Moreover, the difference of the two prefectors $\frac{1}{4} \mathrm{e}^{1 / 2} 2^{2 / 3} A^{-6}=0.1471 \ldots$ and $\pi^{-5 / 2}(1+\pi / 2)=0.1470 \ldots$ is minute, supporting therefore the validity of our approach. 

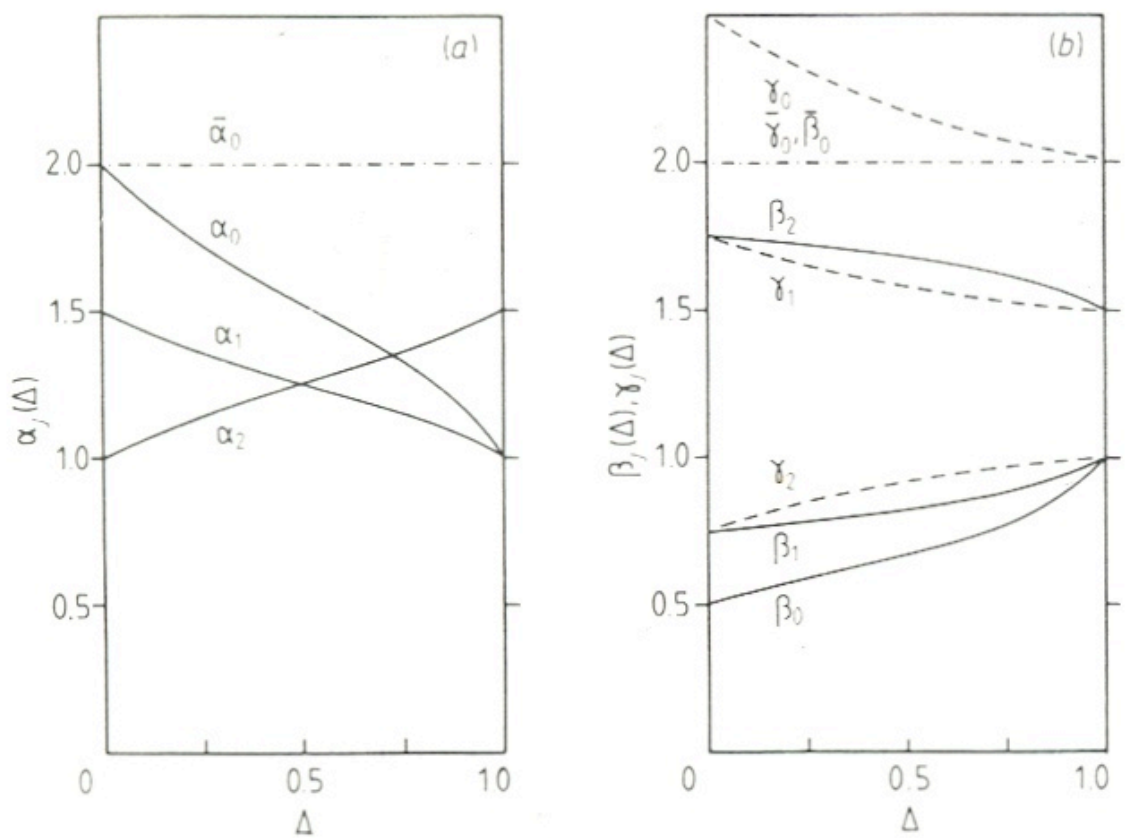

Figure 8. Anisotropy dependence (a) of the exponents $\alpha_{j}$ of equation (3.36b) and (b) of the exponents $\beta_{j}$ and $\gamma_{j}$ (broken lines) of equation (3.37b). The asymptotic behaviour of $\left\langle S_{l}^{\mu}(t) S_{l}^{\mu}\right\rangle$ is dominated by the term with the smallest exponent. The 'normal' exponents f $\bar{\alpha}_{0}=\bar{\beta}_{0}=\bar{\gamma}_{0}=2$ originating from the term of $F_{\mu \mu}(\omega)$ linear in $\omega$ is shown as a chain line.

\subsection{Comparison with continuum theories}

The dynamic structure factor for the planar $s=\frac{1}{2} \mathrm{AF}$ at $T=0$ has already been investigated by Luther and Peschel (1975) in the framework of the (continuum) Luttinger model, and by Fogedby (1978) in a continuum Green function approach. By construction, the validity of their results is restricted to small excitation energies, which occur at $q \simeq 0$ and $q \simeq \pi$. In both continuum approaches, however, the dynamic correlation functions are first obtained in $(x, t)$ space, yielding in the asymptotic region

$$
\begin{aligned}
& \left\langle S^{z}(x, t) S^{z}(0,0)\right\rangle \sim \frac{x^{2}+c^{2} t^{2}}{\left(x^{2}-c^{2} t^{2}\right)^{2}}+\cos (\pi x)\left(x^{2}-c^{2} t^{2}\right)^{-1 / 2 \varphi}, \\
& \left\langle S^{x}(x, t) S^{x}(0,0)\right\rangle \sim \frac{x^{2}+c^{2} t^{2}}{\left(x^{2}-c^{2} t^{2}\right)^{1+\varphi / 2+1 / 2 \varphi}}+\cos (\pi x)\left(x^{2}-c^{2} t^{2}\right)^{-\varphi / 2} .
\end{aligned}
$$

Here $c$ is a renormalised Fermi velocity. The anisotropy dependence of $\varphi$ as obtained for the continuum models agrees to leading order in $\Delta$ with the exact $\Delta$-dependence

$$
\varphi=1-\frac{\vartheta}{\pi}, \quad \cos \vartheta=\Delta
$$

for the lattice model (Luther and Peschel 1975). $S_{\mu \mu}(q, \omega)$ is obtained by Fourier transformation ${ }^{2}$

$$
S_{z z}(q, \omega) \sim \begin{cases}\omega \delta(\omega-c q) & q \gtrsim 0, \\ \theta(\omega-c \bar{q})\left(\omega^{2}-c^{2} \bar{q}^{2}\right)^{1 / 2 \varphi-1} & q \lesssim \pi,\end{cases}
$$

\footnotetext{
${ }^{2}$ Note that (3.45a) differs from the corresponding result of Fogedby (1978) by an extra term. This discrepancy originates in the fact that the Fourier transformation implies integrations across the 'light cone' $x^{2}-c^{2} t^{2}=0$, where all terms of (3.42) are divergent. In the case of the first term in (3.42b) the divergence is so strong that it depends crucially on the path. We have chosen a path in such a way that the Fourier transform satisfies for $\Delta=1(\varphi=1)$ the symmetry requirement $S_{z z} \equiv S_{x x}$ which is already fulfilled by (3.42) before the Fourier transformation.
} 


$$
S_{x x}(q, \omega) \sim \begin{cases}\frac{\theta(\omega-c q)}{\Gamma(\varphi / 2-1 / 2 \varphi-1)}\left[\left(\omega^{2}-c^{2} q^{2}\right)^{\varphi / 2+1 / 2 \varphi-1}+\frac{2 c^{2} q^{2}}{\left(\omega^{2}-c^{2} q^{2}\right)^{2-\varphi / 2-1 / 2 \varphi}}\right] & q \gtrsim 0, \\ \theta(\omega-c \bar{q})\left(\omega^{2}-c^{2} \bar{q}^{2}\right)^{\varphi / 2-1} & q \lesssim \pi .\end{cases}
$$

With (3.43) we can identify the exponents appearing here with the exponents (3.8) and (3.17) used in our SWC approach:

$$
\alpha=1-1 / 2 \varphi, \quad \beta=1-\varphi / 2, \quad \gamma=1-\varphi / 2-1 / 2 \varphi .
$$

Comparison with our SWC results shows the following: For small $q$ the narrow SWC A of (3.9) is obviously very similar to the single branch of (3.44a). Both results have the same frequency moments for $q \ll 1$. For $q \lesssim \pi(3.44 \mathrm{~b})$ yields the same characteristic power-law behaviour as (3.9) without, however, the corresponding structure at higher frequencies. Comparing (3.18) for $q \gtrsim 0$ with (3.45b), we observe that the first term of (3.45a) corresponds to $S_{x x}^{\mathrm{C}}(q, \omega)$ of $(3.18)$, a quantity which vanishes at the lower threshold $\omega=c q$. The second term reflects the contribution of $S_{x x}^{\mathrm{B}}(q, \omega)$ in $(3.18 \mathrm{~b})$ which is infinite at $\omega=c q$ and whose intensity vanishes for $q \rightarrow 0$. In the isotropic limit $(\varphi \rightarrow 1)$, the $\Gamma$-function in the denominator diverges, and only the second term of (3.45a) survives (due to its own divergence at $\omega=c q$ yielding (3.44a)). For $q \lesssim \pi,(3.45 \mathrm{~b})$ is in good agreement with the dominating $S_{x x}^{\mathrm{B}}(q, \omega)$ of (3.18). But a contribution of the form $\omega \delta(\omega-c \bar{q})$ coming from $S_{x x}^{\mathrm{C}}(q, \omega)$ does not appear as a separate term in $(3.45 \mathrm{~b})$. However, the intensity of this contribution vanishes for $\bar{q} \rightarrow 0$ and, moreover, $S_{x x}^{\mathrm{B}}(q, \omega)$ has already a strong singularity at $\omega=c \bar{q}$.

\section{The antiferromagnet with $s>\frac{1}{2}$}

\subsection{Comparison with classical spin-wave theory}

Here we compare the results of Sec. 3 with the predictions of classical spin-wave theory, in order to emphasise the quantum effects inherent in our results. Linear spin-wave theory for the planar AF (2.1) starts from a two-sublattice ground state with spins lying in the $X Y$ plane. In order to restore the rotational symmetry in the $X Y$ plane, we take an average over all orientations of the sub lattice magnetisations. In the reduced zone two branches of normal modes are obtained. For comparison with the quantum $\mathrm{AF}$ it is convenient to use a representation in the extended zone. Here, the normal modes form a single branch, but the assigned $q$ depends on which spin components are considered (see e.g. Muller et al 1981b). We obtain for the OP component $(\mu=z$, upper sign) and for the IP components ( $\mu=x, y$, lower sign) the dispersion (Keffer 1966) ${ }^{3}$

$$
\omega_{\mu}(q)=J[(1 \mp \cos q)(1 \pm \Delta \cos q)]^{1 / 2} .
$$

Zero-point fluctuations can be introduced either via the sum rules (2.8) or via the quantummechanical fluctuation-dissipation theorem from the static susceptibilities $\chi_{\mu \mu}(q)$. The latter are derived directly from the linearised equations of motion corresponding to (2.1) including a perturbing field. Both approaches yield the same results

$$
S_{\mu \mu}(q, \omega)=2 \pi I_{\mu \mu}(q) \delta\left(\omega-\omega_{\mu}(q)\right)
$$

with integrated intensities

$$
I_{z z}(q)=\frac{1}{4}\left(\frac{1-\cos q}{1+\Delta \cos q}\right)^{1 / 2}, \quad I_{x x}(q)=\frac{1}{8}\left(\frac{1-\Delta \cos q}{1+\cos q}\right)^{1 / 2}
$$

The corresponding susceptibilities are

$$
\chi_{z z}(q)=\frac{1}{2 J(1+\Delta \cos q)}, \quad \chi_{x x}(q)=\frac{1}{4 J(1+\cos q)} .
$$

\footnotetext{
${ }^{3}$ Throughout Sec. 4 we normalise excitation energies with respect to $2 s$.
} 
We observe that the OP fluctuations (4.2) in the spin-wave approximation have a gap at $q=\pi$ increasing with planar anisotropy $\epsilon_{\text {Gap }}=[2(1-\Delta)]^{1 / 2}$. This is in marked contrast to the quantum result (3.9) which has gapless excitations both at $q=0$ and $q=\pi$. Note, however, that in the quantum case the spectral weight of $S_{z z}(\pi, \omega)$ is - with increasing anisotropy - also redistributed towards higher energies, but rather in terms of a varying exponent than in terms of an energy gap. Another contrasting feature is the fact that $\chi_{z z}(\pi)$ stays finite for $\Delta<1$ in the classical case, whereas the quantum result (3.26) is divergent for $q \rightarrow \pi$. Also, the type of divergence of $I_{x x}(q)$ and $\chi_{x x}(q)$ at $q=\pi$ differs for $\Delta<1$ from the quantum results $\left(\bar{q}^{-1}\right.$ and $\bar{q}^{-2}$ as compared with $\bar{q}^{1-2 \beta}$ and $\bar{q}^{-2 \beta}$, respectively). The IP component of (4.2) has a gap at $q=0$. The quantum result (3.18), on the other hand, has gapless excitations at the zone centre with intensity vanishing for $q \rightarrow 0\left(S_{x x}^{\mathrm{B}}\right)$. In addition to that, it has excitations with finite intensity for $q \rightarrow 0$ in a broad frequency range. Note also that $\chi_{x x}(q)$ in the spin-wave approximation is independent of $\Delta$, while the corresponding result (3.30) for $s=\frac{1}{2}$ varies with $\Delta$.

\subsection{Isotropic $s \geq \frac{1}{2}$ Heisenberg antiferromagnet}

For a full understanding of the differences between our results for the quantum AF (discussed in Sec. 3) and the prediction of linear spin-wave theory (reviewed in Sec. 4.1), it would be important to know how the classical limit is approached in the quantum treatment as the spin quantum number $s$ goes to infinity. In this section we discuss how in the present approach such a link between the quantum case and the classical limit can be obtained for the isotropic $\operatorname{AF}(\Delta=1)$. For this case, the two-sub lattice theory (Anderson 1952) predicts that the zero-point fluctuations are characterised by a $\delta$-peak at the classical spin-wave frequency

$$
S_{\mu \mu}(q, \omega)=A_{\mathrm{cl}} \tan \frac{q}{2} \delta(\omega-J \sin q) .
$$

Here we have taken an average over all three directions. In the extreme quantum limit $\left(s=\frac{1}{2}\right)$, on the other hand, $S_{\mu \mu}(q, \omega)$ is governed by a whole continuum of excitations according to (3.9), and at first sight it is not obvious how with increasing $s$ the classical limit will be approached. There are two important hints leading to an answer of this problem:

(i) Finite-chain calculations on isotropic systems (2.1) with spin quantum number $s=1$ indicate that still most of the spectral weight of $S_{\mu \mu}(q, \omega)$ is concentrated between two sine-like dispersion branches similar to (3.2). This suggests the presence of a SWC in the thermodynamic limit similar to the case $s=\frac{1}{2}$.

(ii) By use of a Holstein-Primakoff-type approach, Mikeska (1975) investigated quantum effects for $s \gg 1$ in the isotropic AF. He found for the $T=0$ structure function at $q \simeq \pi$ and $\omega \ll J$ to leading order in $1 / s$ the result

$$
S_{\mu \mu}(q, \omega) \propto \theta(\omega-J \sin q)\left(\omega^{2}-J^{2} \sin ^{2} q\right)^{-\tilde{\alpha}}
$$

with an exponent depending on the spin quantum number as

$$
\tilde{\alpha}(s)=1-\frac{1}{\pi s}+\mathrm{O}\left(s^{-2}\right) .
$$

This result (4.6) is highly suggestive since it is - at $q \lesssim \pi, \omega \ll J$ - consistent with an ansatz of the form (3.9) as used for the $s=\frac{1}{2} \mathrm{AF}$. Therefore, we are encouraged to extend (4.6) to the whole Brillouin zone and to higher frequencies, and generalise it for arbitrary spin quantum number $s$. We propose the following analytic expression:

$$
S_{\mu \mu}(q, \omega)=\frac{2 \tilde{A}(s)}{B(1-\tilde{\alpha}, 1 / 2+\tilde{\alpha})} \frac{\theta\left(\omega-\tilde{\epsilon}_{1}(q)\right) \theta\left(\tilde{\epsilon}_{2}(q)-\omega\right)}{\left[\omega^{2}-\tilde{\epsilon}_{1}^{2}(q)\right]^{\tilde{\alpha}}\left[\tilde{\epsilon}_{2}^{2}(q)-\omega^{2}\right]^{1 / 2-\tilde{\alpha}}} .
$$

The continuum boundaries are expected to be given by

$$
\tilde{\epsilon}_{1}(q)=\frac{1}{2} p(s) \sin q, \quad \tilde{\epsilon}_{2}(q)=p(s) \sin \frac{q}{2},
$$


where the amplitude $p(s)$ is required to take the value $\pi J$ in the $s=\frac{1}{2}$ limit in agreement with (3.2) and will approach the (normalised) classical spin-wave result $p(s) \rightarrow 2 J$ for large $s$. In the classical limit the exponent $\tilde{\alpha}(s)$ becomes unity according to (4.7) whereas comparison of (4.8) with (3.9) for $\Delta=1$ makes clear that for $s=\frac{1}{2}$ the exponent should extrapolate to $\tilde{\alpha}=\frac{1}{2}$. The prefactor $\tilde{A}(s)$ will be determined below. We again use the beta function as normalising factor, which becomes crucial in the limit $s \rightarrow \infty$. The validity of the ansatz (4.8) is supported by the fact that it satisfies the sum rule (2.8). This is a very strong requirement. In fact, the first frequency moment of (4.8)

$$
K_{\mu \mu}(q)=\frac{\tilde{A} p}{4 \pi}(1-\cos q)
$$

reproduces the correct q-dependence of $(2.9 \mathrm{~b})$, and $\tilde{A}(s)$ is determined by comparing the prefactors of $(2.9 \mathrm{~b})$ and $(4.10)$ as to be

$$
\tilde{A}(s)=-\frac{8 \pi J}{p(s)} F_{\mu}(s) .
$$

For the isotropic AF the NN correlation functions $F_{\mu}$ are related - with the appropriate normalisation - to the ground-state energy by $F_{\mu}(s)=\frac{1}{3} E_{\mathrm{G}}(s) / 4 s^{2} J N, \mu=x, y, z$. The $s=\frac{1}{2}$ result has been given in Sec. 3.1. Linear spin-wave theory with zero-point fluctuations taken into account (Anderson 1952) yield to order $1 / s$

$$
F_{\mu}(s)=-\frac{1}{12}[1+(1-2 / \pi) / s], \quad s \gg 1 .
$$

In the limit $s \rightarrow \infty$ these $F_{\mu}$ reflect the result for the Néel ground state averaged over all directions in spin space. By comparing successive frequency moments it can be shown that for $s \rightarrow \infty, \tilde{\alpha} \rightarrow 1$ the two results (4.5) and (4.8) are equivalent (with $A_{\mathrm{cl}}=\pi / 3$ ). In this limit, the divergence in (4.8) at $\tilde{\epsilon}_{1}(q)$ becomes very strong (being no longer integrable with respect to $\omega$ ). Due to the diverging beta function, on the other hand, the normalisation leaves nonzero intensity only at $\tilde{\epsilon}_{1}(q)$. Thus, our ansatz (4.8), which was originally constructed for an $s=\frac{1}{2}$ system, leads in the limit $s \rightarrow \infty$ to the exact classical spin-wave result (4.5) in a straightforward manner.

Figure 9 shows $S_{\mu \mu}(q, \omega)$ evaluated from (4.8) at fixed $q=4 \pi / 5$ as a function of $\omega$ for $s=\frac{1}{2}, 1, \frac{5}{2}$. It illustrates how the Iineshape asymmetry becomes less and less pronounced as the spin quantum number increases. In the limit $s \rightarrow \infty$ it disappears completely. Owing to finite-temperature effects

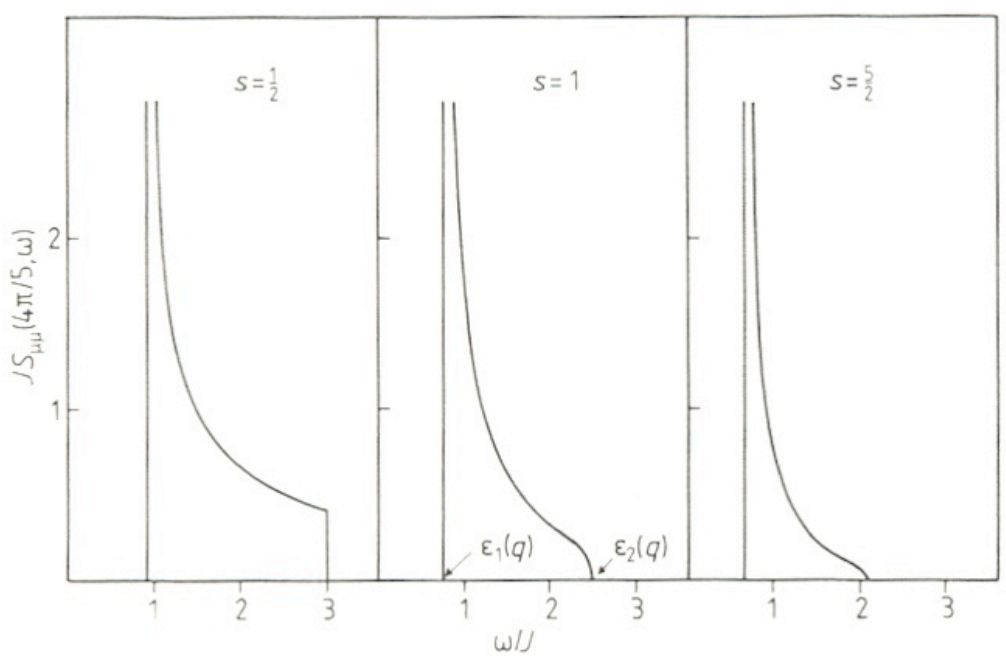

Figure 9. Dynamic structure factor $S_{\mu \mu}(q, \omega)$ of equation (4.8) versus $\omega$ at fixed $q=4 \pi / 5$ of the isotropic Heisenberg AF with spin quantum numbers $s=\frac{1}{2}, 1, \frac{5}{2}$. 
which generally produce a broadening of the spin-wave peaks, asymmetric lineshapes in inelastic neutron scattering have so far been detected only for $s=\frac{1}{2}$ compounds (Heilmann et aI1978). However, if one looks at the early neutron scattering data on the $s=\frac{5}{2} \mathrm{AF}$ compound TMMc as obtained by Hutchings et at (1972) one is inclined to observe at low $T$ a slight asymmetry in the peaks for wave numbers close to the zone boundary (see figure 11 of their paper), though it has not been interpreted by the authors as a physical effect. We should like, therefore, to call the attention of experimentalists who are currently investigating dynamical properties of TMMC to this effect.

The integrated intensity of (4.8) has the same structure as (3.21). For $s=\frac{1}{2}\left(\tilde{\alpha}=\frac{1}{2}\right)$ it coincides with (3.22), and for $s=\infty(\tilde{\alpha}=1)$ it yields $I_{\mu \mu}(q)=\tilde{A} \tan (q / 2)$ in accordance with (4.3). In real space the spin correlations behave asymptotically as $\left\langle S_{l}^{\mu} S_{l+R}^{\mu}\right\rangle \sim(-1)^{R} / R^{2(1-\tilde{\alpha})}$. We observe that the power-law decay for finite $s$ changes into true antiferromagnetic LRO in the classical limit $\tilde{\alpha} \rightarrow 1$. The $q$-dependent susceptibility of (4.8) is obtained as

$$
\chi_{\mu \mu}(q)=\frac{\tilde{A}(s)}{\pi p(s)}{ }_{2} F_{1}\left(1, \tilde{\alpha}+\frac{1}{2} ; \frac{3}{2} ; \sin ^{2} \frac{q}{2}\right) .
$$

For $s \rightarrow \infty$ it reduces to $\chi_{\mu \mu}(q)=(\tilde{A} / \pi J)(1+\cos q)^{-1}$ in agreement with (4.4). The low-frequency behaviour of the autocorrelation function calculated from (4.8) displays a power-law divergence

$$
F_{\mu \mu}(\omega) \sim \omega^{1-2 \tilde{\alpha}}, \quad \omega \ll J ; \quad s>\frac{1}{2}
$$

except for $s=\frac{1}{2}$ where it stays finite for $\omega \rightarrow 0$ according to (3.34). This qualitatively different behaviour for $s=\frac{1}{2}$ and $s>\frac{1}{2}$ has shown up in several low- $T$ spin-lattice relaxation measurements lacking, however, a satisfactory explanation. By various investigations on different $s=\frac{1}{2} \mathrm{AF}$ compounds it has been found that the relaxation time $T_{2}$ at small magnetic fields and low $T$ has only a very weak temperature dependence (Ehrenfreund et al 1973, Azevedo et al 1979, Groen et al 1978, 1979, 1980). For the $s=\frac{5}{2}$ AF TMMC, on the other hand, Richards and Borsa (1974) and Hone et al (1974) have observed that $T_{1}$ has a very strong temperature dependence in agreement with a diverging $F_{\mu \mu}(\omega=0)$ for $T \rightarrow 0$. In view of the increasing number of available quasi-1D $\mathrm{AF}$ compounds with different spin quantum numbers it would be interesting to investigate such $s$-dependent effects more systematically.

\subsection{Outlook}

The Hamiltonian (2.1) can be understood as depending on the two parameters $\Delta$ (planar anisotropy, $0 \leq \Delta \leq 1$ ) and $s$ (spin quantum number, $\frac{1}{2} \leq s \leq \infty$ ). We can represent their range in the two-dimensional parameter space in the form of a rectangle. We ignore here the fact that $s$ is actually a discrete variable. In Secs. 3 and 4 we have presented detailed results of $S_{\mu \mu}(q, \omega)$ for parameters on three of the four edges of the rectangle:

$$
s=\frac{1}{2}, 0 \leq \Delta \leq 1 ; \quad s=\infty, 0 \leq \Delta \leq 1 ; \quad \Delta=1, \frac{1}{2} \leq s \leq \infty .
$$

Now it would be highly interesting to 'fill in' the rectangle, i.e. to provide equivalent results for the fourth edge and the interior. To the best of our knowledge there are practically no such results available in the literature. The present analytic approach cannot easily be generalised to arbitrary $(\Delta, s)$, because it relies on the knowledge of the energies of the relevant excitations and behaviour of their matrix elements (2.11). Such information is presently not available. Therefore we indicate here a qualitative way to extend our results phenomenologically into the rectangle by making the following conjectures (e.g. for the OP fluctuations):

(i) $S_{z z}(q, \omega)$ is always governed by a two-parameter SWC between a lower boundary $\epsilon_{1}(q, \Delta, s)$ and an upper boundary $\epsilon_{2}(q, \Delta, s)$.

(ii) $S_{z z}(q, \omega)$ can be represented by analytic expression of the form (3.9) involving new $(\Delta, s)$ dependent boundaries and an exponent $\alpha$ which also depends on both $\Delta$ and $s$. 
(iii) The quantities $\alpha, \epsilon_{1}, \epsilon_{2}$ which are known on three edges of the rectangle continue in some smooth way into the interior. Let us discuss briefly the implications of these assumptions. We restrict ourselves here to the qualitative features. The dispersion curve $\epsilon_{1}(q, \Delta, s)$ of the lowest excitations contributing to $S_{z z}(q, \omega)$ is a sine curve for $\left(0 \leq \Delta \leq 1, s=\frac{1}{2}\right)$ and $(\Delta=1,0 \leq s \leq \infty)$ i.e. gap less at $q=\pi$. Along the line $(0 \leq \Delta \leq 1, s=\infty))$ we know that it has a gap at $q=\pi$ of magnitude $\Gamma(\Delta, \infty)=J[2(1-\Delta)]^{1 / 2}$. The above continuity assumption then suggests that within the rectangle $\Gamma(\Delta, s)$ behaves qualitatively as indicated in figure 10(a). It appears that gapless excitations at $q=\pi$ are really restricted to $s=\frac{1}{2}$ or $\Delta=1$. Figure 10(b) shows qualitatively how we expect the exponent $\alpha$ to vary throughout the rectangle. Here we should remark that a quantitative analysis shows that the phenomenological extension of the ansatz (3.9) to the limit $(0 \leq \Delta \leq 1, s \rightarrow \infty)$ reproduces exactly the prediction of classical spin-wave theory (4.2).
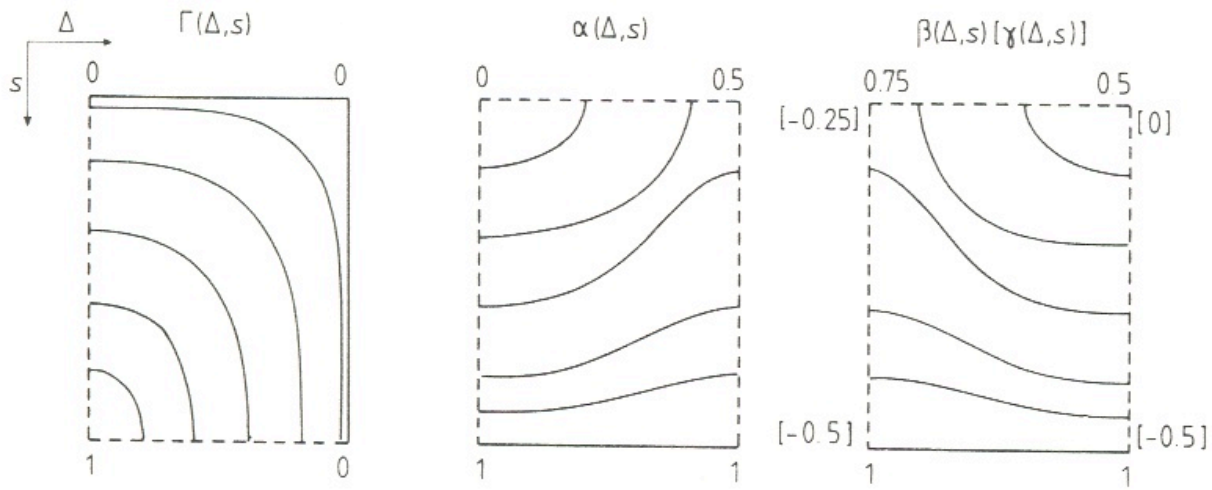

Figure 10. Qualitative picture in the two-dimensional parameter-space $(\Delta, s)$ of (a) lines of constant gap energy and (b), (c) lines of constant values of the exponents $\alpha, \beta, \gamma$.

What does our theory predict for the weakly planar $(\Delta=0.984) s=\frac{5}{2}$ AF TMMC? From figure 10(a) we conclude that the spectrum must have a gap at $q=\pi$. Figure $10(\mathrm{~b})$, on the other hand suggests that $\alpha>\frac{1}{2}$ for TMMC. This implies that an expression of the form (3.9) has only a divergence at the lower boundary $\epsilon_{1}(q, \Delta, s)$. Therefore, we expect for TMMC that $S_{z z}(q, \omega)$ is characterised by a single peak at an energy which has a small gap at $q=\pi$. That is indeed what has been observed in recent inelastic neutron scattering experiments (Heilmann et al 1979). We should point out, however, that this behaviour is quite different from what we predict for a corresponding $s=\frac{1}{2}$ planar AF; see figure 4). In an analogous way the IP structure factor of the form (3.18) may be extended to arbitrary $(\Delta, s)$ in the rectangle. The gap of magnitude $\Gamma(\Delta, s)$ is expected here to show up at $q=0$. Figure 10 (c) shows the expected variation of the exponents $\beta, \gamma$.

In summary, the present work has provided a broad understanding of the $T=0$ dynamics of the 1D planar antiferromagnet. In Sec. 3 we have found by some new approach approximate analytic expressions for both the OP and the IP dynamic structure factors, and have discussed their implications in relation to various interesting theoretical features as well as to possible experimental observations. In Sec. 4 we have established a link to the predictions of a classical linear spin-wave theory, which are expected to be valid in the limit $s \rightarrow \infty$. The considerations for $s>\frac{1}{2}$ are kept rather qualitative. Further investigations are necessary in order to put the theory in this regime onto a solid basis. We hope that this work will stimulate further (dynamical) experimental investigations on quasi-1D magnetic compounds.

\section{Acknowledgments}

The authors are indebted to Professors J C Bonner and H W Capel for many valuable comments and stimulating discussions. This work was supported by the Swiss National Science Foundation. We have used a modified cmpj.sty style file. 


\section{References}

Anderson P W 1952 Phys. Rev. 86694

Azevedo L J, Narath A, Richards P M and Soos Z G 1979 Phys. Rev. Lett. 43875

Baxter R J 1972 Ann, Phys., NY 70323

Bethe H 1931 Z. Phys. 71205

des Cloizeaux J and Gaudin M 1961. Math. Phys. 71384

Ehrenfreund E, Rybaczewski E F, Garito A F, Heeger A J and Pincus P 1973 Phys. Rev. B 7421

Fogedby H C 1978 J. Phys. C: Solid State Phys. 114767

Fowler M and Puga M W 1979 Phys. Rev. B 195906

Gradshteyn I Sand Ryzhik I M 1965 Tables of Integrals, Series and Products (New York: Academic Press)

Griffiths R B 1964 Phys. Rev. 133 A768

Groen J P, Capel H W, Perk J H H, Klaassen T O and Poulis N J 1979 Physica 97B 126

Groen J P, Klaassen T O and Poulis N J 1978 Phys. Lett. 68A 381

Groen J P, Klaassen T O, Poulis N J, Müller G, Thomas H and Beck H 1980 Phys. Rev. B 225369

Heilman I U, Birgenau R J, Endoh Y, Reiter G, Shirane G and Holt S L 1979 Solid State Commun.

31607

Heilmann I U, Shirane G, Endoh Y, Birgenau R J and Holt S L 1978 Phys. Rev. B 183530

Hohenberg P C and Brinkman W F 1974 Phys. Rev. B 10128

Hone D, Scherer C and Borsa F 1974 Phys, Rev. B 9965

Hutchings M T, Shirane G, Birgenau R J and Holt S L 1972 Phys. Rev. B 51999

Ishimura Nand Shiba H 1977 Prog. Theor. Phys. 571862

- 1980 Prog. Theor. Phys. 63743

Johnson J D, Krinsky S, McCoy B M 1973 Phys. Rev. A 82526

Katsura S, Horiguchi T and Suzuki M 1970 Physica 4667

Keffer F 1966 Handb. Phys. ed S Flügge (Berlin: Springer-Verlag) vol. XVIII/2

Lieb E H and Mattis D C 1962], Math, Phys. 3749

Lieb E H, Schultz T and Mattis D C 1961 Ann. Phys., NY 16407

Luther A and Peschel I 1975 Phys. Rev. B 123908

Marshall Wand Lovesey S W 1971 Theory of Thermal Neutron Scattering (Oxford: Clarendon)

Mattis D C 1979 Phys. Rev. Lett. 421503

Mikeska H J 1975 Phys. Rev. B 122794

Müller G and Beck H 1978 J. Phys. C: Solid State Phys. 11483

Müller G, Beck H and Bonner J C 1979 Phys, Rev. Lett, 4375

Müller G, Thomas H and Beck H 1980 Ordering in Strongly Fluctuating Condensed Matter Systems ed T Riste (New York: Plenum) p 151

Müller G, Thomas H, Beck H and Bonner J C 1981a Solid State Commun, 381

- 1981 Phys. Rev. B in press

Niemeijer T 1967 Physica 36377

Richards P M and Borsa F 1974 Solid State Commun. 15135

Vaidya H G and Tracy C A 1978 Physica 92A 1

Yang C N and Yang C P 1966 Phys. Rev. 150327 